\title{
SHORTENING BINARY COMPLEXES AND COMMUTATIVITY OF K-THEORY WITH INFINITE PRODUCTS
}

\author{
DANIEL KASPROWSKI AND CHRISTOPH WINGES
}

\begin{abstract}
We show that in Grayson's model of higher algebraic K-theory using binary acyclic complexes, the complexes of length two suffice to generate the whole group. Moreover, we prove that the comparison map from Nenashev's model for $K_{1}$ to Grayson's model for $K_{1}$ is an isomorphism. It follows that algebraic $K$-theory of exact categories commutes with infinite products.
\end{abstract}

\section{INTRODUCTION}

On a conceptual level, the algebraic $K$-theory functor is by now well understood in terms of a universal property, which encapsulates the known fundamental properties of Quillen's or Waldhausen's construction [Bar16, BGT13].

One of the more elusive properties of algebraic $K$-theory is its compatibility with infinite products. This question was studied by Carlsson [Car95] in connection to work of Carlsson-Pedersen on the split injectivity of the $K$-theoretic assembly map [CP95, and permeates the literature adapting their "descent" argument to prove more general cases of the $K$-theoretic Novikov conjecture [BR07, RTY14, Kas15]. Carlsson's proof, while relying on the Additivity theorem, is for the most part concerned with simplicial techniques involving what he calls quasi-Kan complexes.

The present article aims to provide a different perspective on the question. In Gra12, Grayson showed that the higher algebraic $K$-theory of an exact category can be expressed in terms of binary acyclic complexes. See Section 2 for a quick review.

In [Nen98] Nenashev gave a different presentation $K_{1}^{N}(\mathcal{N})$ of $K_{1}(\mathcal{N})$ whose generators are binary acyclic complexes of length two. Regarding a binary acyclic complex of length two as a class in $K_{0}(\Omega \mathcal{N})$ defines a natural homomorphism

$$
\Phi: K_{1}^{N}(\mathcal{N}) \rightarrow K_{0}(\Omega \mathcal{N}) ;
$$

see [Gra12, Remark 8.1] and the beginning of Section 5.

Unpublished work of Grayson shows that $\Phi$ is a surjection, cf. Gra12, Remark 8.1]. Building on Grayson's unpublished argument (see Remark 5.5), we improve this to a bijectivity statement.

Theorem 1.1. The map $\Phi$ is an isomorphism.

Received by the editors June 14, 2017, and, in revised form, May 31, 2019.

2010 Mathematics Subject Classification. Primary 19D06; Secondary 18E10.

Key words and phrases. Shortening, binary acyclic complexes, algebraic K-theory of infinite products.

Both authors were funded by the Deutsche Forschungsgemeinschaft (DFG, German Research Foundation) under Germany's Excellence Strategy - GZ 2047/1, Projekt-ID 390685813.

The second author was furthermore supported by the Max Planck Society and Wolfgang Lück's ERC Advanced Grant "KL2MG-interactions" (no. 662400). 
We use this to show the following theorem.

Theorem 1.2. For every family $\left\{\mathcal{N}_{i}\right\}_{i \in I}$ of exact categories, the natural map

$$
\mathbb{K}^{-\infty}\left(\prod_{i \in I} \mathcal{N}_{i}\right) \rightarrow \prod_{i \in I} \mathbb{K}^{-\infty}\left(\mathcal{N}_{i}\right)
$$

is a $\pi_{*}$-isomorphism. Here $\mathbb{K}^{-\infty}$ denotes non-connective algebraic $K$-theory.

Since Grayson's results in Gra12 rely only on the fundamental properties of $K$-theory, our proof is not only elementary, but also exhibits Theorem 1.2 as a consequence of the universal property of algebraic $K$-theory.

As a corollary of Theorem 1.1, we obtain the following theorem.

Theorem 1.3. The canonical map $K_{0}\left(\Omega_{[0,2]}^{n} \mathcal{N}\right) \rightarrow K_{0}\left(\Omega^{n} \mathcal{N}\right)$ is a surjection.

We also obtain the following theorem.

Theorem 1.4. For every $n \in \mathbb{N}$ the canonical map $K_{0}\left(\Omega_{[0,3]}^{n} \mathcal{N}\right) \rightarrow K_{0}\left(\Omega^{n} \mathcal{N}\right) \cong$ $K_{n}(\mathcal{N})$ admits a natural section.

The existence of a section to $K_{0}\left(\Omega_{[0,4]}^{n} \mathcal{N}\right)$ is a direct consequence of Theorem 1.1. but our proof actually implies this stronger statement.

Since the proof of Theorem 1.1 is technical, we will begin by showing versions of Theorems 1.3 and 1.4 in Section 3. Here the proofs are considerably easier and they suffice to deduce Theorem 1.2. In Section 4, we use the right inverse to show Theorem 1.2 Finally, we give the proof of Theorem 1.1 in Section 5

The results were adapted to the setting of stable $\infty$-categories in KW19.

\section{BinARY COMPLEXES}

In this section, we give a quick review of Grayson's description of the higher algebraic $K$-groups Gra12. In the following $\mathcal{N}$ will always denote an exact category. Chain complexes in $\mathcal{N}$ will always be assumed to be bounded. Denote by $C \mathcal{N}$ the category of (bounded) chain complexes in $\mathcal{N}$.

Definition 2.1. A chain complex $\left(P_{*}, d\right)$ in $\mathcal{N}$ is called acyclic if each differential admits a factorization into an admissible epimorphism followed by an admissible monomorphism

$$
d_{n}: P_{n} \rightarrow J_{n-1} \longmapsto P_{n-1}
$$

such that $J_{n} \longmapsto P_{n} \rightarrow J_{n-1}$ is a short exact sequence.

Denote by $C^{q} \mathcal{N} \subseteq C \mathcal{N}$ the full subcategory of acyclic chain complexes.

Definition 2.2. A binary acyclic complex $\left(P_{*}, d, d^{\prime}\right)$ is a graded object $P_{*}$ over $\mathcal{N}$ together with two degree -1 maps $d, d^{\prime}: P_{*} \rightarrow P_{*}$ such that both $\left(P_{*}, d\right)$ and $\left(P_{*}, d^{\prime}\right)$ are acyclic chain complexes. The differentials $d$ and $d^{\prime}$ are called the top and bottom differential.

A morphism of binary acyclic complexes is a degree 0 map of underlying graded objects which is a chain map with respect to both differentials. The resulting category of binary acyclic complexes is denoted by $B^{q} \mathcal{N}$. There is a natural exact functor $\Delta: C^{q}(\mathcal{N}) \rightarrow B^{q}(\mathcal{N})$ which duplicates the differential of a given acyclic chain complex. 
Fix $n>0$. Since both $C^{q} \mathcal{N}$ and $B^{q} \mathcal{N}$ are exact categories, these constructions can be iterated. For any finite sequence $\mathbb{W}=\left(W_{1}, \ldots, W_{n}\right)$ in $\{B, C\}$, denote by $\mathbb{W}^{q} \mathcal{N}$ the category $W_{1}^{q} \ldots W_{n}^{q} \mathcal{N}$. If $\mathbb{W}$ is the constant sequence on the letter $B$, we also write $\left(B^{q}\right)^{n} \mathcal{N}$. Letting $\mathbb{W}$ vary over all possible choices defines a commutative $n$-cube of exact categories which induces a commutative $n$-cube of spectra upon taking algebraic $K$-theory. The spectrum $\mathbb{K}\left(\Omega^{n} \mathcal{N}\right)$ is defined to be the total homotopy cofiber of this cube.

We rely on the following result about $\mathbb{K}\left(\Omega^{n} \mathcal{N}\right)$.

Theorem 2.3 (Grayson, Gra12, Corollary 7.2]). The abelian groups $K_{n}(\mathcal{N})$ and $K_{0}\left(\Omega^{n} \mathcal{N}\right)$ are naturally isomorphic.

This theorem facilitates a completely algebraic description of higher $K$-theory Gra12, Corollary 7.4]. For example, it implies that $K_{1}(\mathcal{N})$ can be described as the Grothendieck group of the category of binary acyclic complexes $B^{q} \mathcal{N}$ with the additional relation that a binary acyclic complex represents the trivial class if its top and bottom differential coincide. We use this description of $K_{1}(\mathcal{N})$ extensively in Section 3.

Throughout this article, we employ the following variations of this construction: Let $J \subseteq \mathbb{Z}$ be an interval, i.e., $J=\{z \in \mathbb{Z} \mid a \leq z \leq b\}$ for some $a, b \in \mathbb{Z} \cup\{ \pm \infty\}$. Then we denote by $B_{J}^{q} \mathcal{N}$ and $C_{J}^{q} \mathcal{N}$ the categories of (binary) acyclic complexes supported on $J$. Thus, any sequence of intervals $\mathbb{J}=\left(J_{1}, \ldots, J_{n}\right)$ in $\mathbb{Z}$ gives rise to an abelian group $K_{0}\left(\Omega_{\mathbb{J}} \mathcal{N}\right):=K_{0}\left(\Omega_{J_{1}} \ldots \Omega_{J_{n}} \mathcal{N}\right)$. If $\mathbb{J}^{\prime}=\left(J_{1}^{\prime}, \ldots, J_{n}^{\prime}\right)$ is another such sequence satisfying $J_{k} \subseteq J_{k}^{\prime}$ for all $k$, we have a natural homomorphism

$$
i_{\mathbb{J}, \mathbb{J}^{\prime}}: K_{0}\left(\Omega_{\mathbb{J}} \mathcal{N}\right) \rightarrow K_{0}\left(\Omega_{\mathbb{J}^{\prime}} \mathcal{N}\right) \text {. }
$$

Note that $\Delta: C_{J}^{q} \mathcal{N} \rightarrow B_{J}^{q} \mathcal{N}$ admits two natural splits $\top$ and $\perp$ which forget the bottom, respectively, top, differential of a binary acyclic complex. Using one of these, we see that $i_{\mathbb{J}, \mathbb{J}^{\prime}}$ is naturally a retract of the homomorphism

$$
K_{0}\left(\Omega_{J_{1}} B_{J_{2}}^{q} \ldots B_{J_{n}}^{q} \mathcal{N}\right) \rightarrow K_{0}\left(\Omega_{J_{1}^{\prime}} B_{J_{2}^{\prime}}^{q} \ldots B_{J_{n}^{\prime}}^{q} \mathcal{N}\right) .
$$

Moreover, we observe that any permutation $\sigma:\{1, \ldots, n\} \stackrel{\cong}{\longrightarrow}\{1, \ldots, n\}$ induces an isomorphism

$$
K_{0}\left(\Omega_{I_{1}} \ldots \Omega_{I_{n}} \mathcal{N}\right) \cong K_{0}\left(\Omega_{I_{\sigma}(1)} \ldots \Omega_{I_{\sigma}(n)} \mathcal{N}\right) .
$$

It is notationally convenient to work with $\mathbb{N}$-graded bounded chain complexes instead of $\mathbb{Z}$-graded chain complexes. The following lemma justifies this convention.

Lemma 2.4. The natural map $K_{0}\left(\Omega_{[0, \infty)}^{n} \mathcal{N}\right) \rightarrow K_{0}\left(\Omega^{n} \mathcal{N}\right)$ is an isomorphism for all $n \geq 1$.

Proof. We begin with the case $n=1$. The map $K_{0}\left(\Omega_{[0, \infty)} \mathcal{N}\right) \rightarrow K_{0}(\Omega \mathcal{N})$ is an isomorphism since the class group of a filtered union is isomorphic to the colimit of the class groups and shifting induces an isomorphism in $K$-theory.

We will now prove the lemma by induction. Assume that it holds for $n-1$. The map $K_{0}\left(\Omega_{[0, \infty)}^{n} \mathcal{N}\right) \rightarrow K_{0}\left(\Omega_{[0, \infty)}^{n-1} \mathcal{N}\right)$ is a retract of $K_{0}\left(\Omega_{[0, \infty)}\left(B_{[0, \infty)}^{q}\right)^{n-1} \mathcal{N}\right) \rightarrow$ $K_{0}\left(\Omega\left(B_{[0, \infty)}^{q}\right)^{n-1} \mathcal{N}\right)$, which is an isomorphism by the induction beginning. Hence, $K_{0}\left(\Omega_{[0, \infty)}^{n} \mathcal{N}\right) \rightarrow K_{0}\left(\Omega \Omega_{[0, \infty)}^{n-1} \mathcal{N}\right)$ is an isomorphism as well. Using that $\Omega$ and $\Omega_{[0, \infty)}$ commute, it suffices to show that $K_{0}\left(\Omega_{[0, \infty)}^{n-1} \Omega \mathcal{N}\right) \rightarrow K_{0}\left(\Omega^{n} \mathcal{N}\right)$ is an isomorphism. This map is a retract of $K_{0}\left(\Omega_{[0, \infty)}^{n-1} B^{q} \mathcal{N}\right) \rightarrow K_{0}\left(\Omega^{n-1} B^{q} \mathcal{N}\right)$, which is an isomorphism by assumption. 
From now on, we write $K_{0}(\Omega \mathcal{N})$ for $K_{0}\left(\Omega_{[0, \infty)} \mathcal{N}\right)$. All chain complexes considered in what follows will be assumed to be positive.

In the remainder of this section, we record some important properties of $K_{0}(\Omega \mathcal{N})$.

Definition 2.5. Let $\mathbb{P}=\left(P_{*}, d, d^{\prime}\right)$ be a binary acyclic complex and let $i \in \mathbb{N}$.

(1) The ith shift $\mathbb{P}[i]$ is defined to be the binary acyclic complex with underlying graded object $P[i]_{*}=P_{*-i}$ and differentials

$$
P[i]_{n}=P_{n-i} \stackrel{d_{n-i}}{\underset{d_{n-i}^{\prime}}{\rightrightarrows}} P_{n-i-1}=P[i]_{n-1} .
$$

(2) The ith suspension $\Sigma^{i} \mathbb{P}$ is defined to be the binary acyclic complex with underlying graded object $\Sigma^{i} P_{*}=P_{*-i}$ and differentials

$$
\Sigma^{i} P_{n}=P_{n-i} \underset{(-1)^{i} d_{n-i}^{\prime}}{\stackrel{(-1)^{i} d_{n-i}}{\longrightarrow}} P_{n-i-1}=\Sigma^{i} P_{n-1} .
$$

Remark 2.6. Our terminology is in disagreement with Gra12, where the suspension is called a shift.

As for ordinary chain complexes, we have the following lemma.

Lemma 2.7 (cf. Gra, Lemma 6.1] and [Har15, Lemma 2.5]). Let $\mathbb{P}$ be a binary acyclic complex supported on $[0, k]$. Then

$$
[\mathbb{P}[i]]=\left[\Sigma^{i} \mathbb{P}\right]=(-1)^{i}[\mathbb{P}] \in K_{0}\left(\Omega_{[0, k+i]} \mathcal{N}\right) .
$$

Proof. The first equality holds since $\mathbb{P}[1] \cong \Sigma \mathbb{P}$. The second equality holds since $\mathbb{P}$ and $\Sigma \mathbb{P}$ fit into a short exact sequence with the cone of $\mathbb{P}$.

Definition 2.8. A binary double complex is a bounded bigraded object $\left(P_{k, l}\right)_{k, l \in \mathbb{N}}$ in $\mathcal{N}$ together with morphisms

$$
d_{k, l}^{h}: P_{k, l} \rightarrow P_{k-1, l}, \quad d_{k, l}^{v}: P_{k, l} \rightarrow P_{k, l-1},
$$

and

$$
d_{k, l}^{\prime, h}: P_{k, l} \rightarrow P_{k-1, l}, \quad d_{k, l}^{\prime, v}: P_{k, l} \rightarrow P_{k, l-1},
$$

such that $\left(P_{*, *}, d^{h}, d^{v}\right)$ and $\left(P_{*, *}, d^{\prime, h}, d^{\prime, v}\right)$ are double complexes in the sense that $\left(P_{*, l}, d^{h}\right)$ and $\left(P_{*, l}, d^{\prime, h}\right)$ are chain complexes for all $l,\left(P_{k, *}, d^{v}\right)$ and $\left(P_{k, *}, d^{\prime, v}\right)$ are chain complexes for all $k$, and $d^{h} d^{v}=d^{v} d^{h}$, respectively, $d^{\prime, h} d^{\prime, v}=d^{\prime, v} d^{\prime, h}$.

We call $\left(P_{*, *}, d^{h}, d^{v}, d^{\prime, h}, d^{\prime, v}\right)$ a binary acyclic double complex if $\left(P_{*, l}, d^{h}, d^{\prime, h}\right)$ is a binary acyclic complex for all $l$ and $\left(P_{k, *}, d^{v}, d^{\prime, v}\right)$ is a binary acyclic complex for all $k$.

Let $\left(P_{*, *}, d^{h}, d^{v}, d^{\prime, h}, d^{\prime, v}\right)$ be a binary acyclic double complex. Forming the total complex of $\left(P_{*, *}, d^{h}, d^{v}\right)$ and $\left(P_{*, *}, d^{\prime, h}, d^{\prime, v}\right)$, using the usual sign trick, produces a binary acyclic complex $\mathbb{T}$. Filtering $\mathbb{T}$ according to the horizontal (respectively, vertical) filtration of the double complexes and applying Lemma 2.7 immediately gives the following lemma.

Lemma 2.9 (Nenashev's relation, cf. Gra12, Remark 8.1] and [Har15, Proposition 2.10]). Let $\left(P_{*, *}, d^{h}, d^{v}, d^{\prime, h}, d^{\prime, v}\right)$ be a binary acyclic double complex. Then we have

$$
\sum_{l}(-1)^{l}\left[P_{*, l}, d^{h}, d^{\prime, h}\right]=\sum_{k}(-1)^{k}\left[P_{k, *}, d^{v}, d^{\prime, v}\right]
$$

in $K_{0}\left(\Omega_{\mathrm{supp}} \mathbb{T} \mathcal{N}\right)$. 
This relation is analogous to the relation used by Nenashev Nen98 to define $K_{1}^{N}(\mathcal{N})$, hence its name.

Remark 2.10. Specifying a binary double complex involves a sizeable amount of data. In order to write down such complexes without occupying too much space, we will follow Nenashev's convention and depict binary double complexes by diagrams of the form

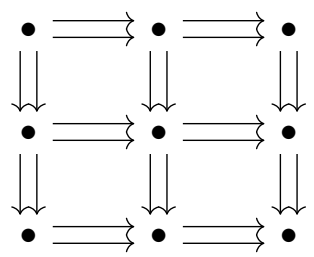

where it is understood that the left vertical morphisms commute with the top horizontal morphisms (corresponding to $d^{h}$ and $d^{v}$ ), and that the right vertical morphisms commute with the bottom horizontal morphisms (corresponding to $d^{\prime}, h$ and $\left.d^{\prime, v}\right)$.

Let $J$ be an object in $\mathcal{N}$ and denote by

$$
\tau_{J}:=\left(\begin{array}{cc}
0 & \operatorname{id}_{J} \\
\operatorname{id}_{J} & 0
\end{array}\right): J \oplus J \rightarrow J \oplus J
$$

the automorphism which switches the two summands.

Lemma 2.11 ([Har15, Proof of Lemma 2.17]). Let $f, g$ be two automorphisms of $P \in \mathcal{N}$. Then

$$
[P \underset{g f}{\stackrel{\mathrm{id}}{\longrightarrow}} P]=[P \underset{f}{\stackrel{\mathrm{id}}{\longrightarrow}} P]+[P \underset{g}{\stackrel{\mathrm{id}}{\longrightarrow}} P] \in K_{0}\left(\Omega_{[0,2]} \mathcal{N}\right) .
$$

In particular, the element

$$
\left[J \oplus J \underset{\tau_{J}}{\stackrel{\mathrm{id}}{\longrightarrow}} J \oplus J\right] \in K_{0}\left(\Omega_{[0,2]} \mathcal{N}\right)
$$

has order at most two.

Proof. Apply Lemma 2.9 to the binary acyclic double complex

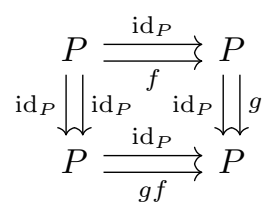

\section{Shortening BINARY COMPLEXES}

The goal of this section is to prove the following weaker versions of Theorems 1.3 and 1.4. These suffice to prove Theorem 1.2 without using the more technical proof of Theorem 1.1. It is also possible to only read Lemma 3.4 and skip the rest of this section, continue with Section [5] and use the splitting obtained there for Section 4

Theorem 3.1. The canonical map $K_{0}\left(\Omega_{[0,4]} \mathcal{N}\right) \rightarrow K_{0}(\Omega \mathcal{N})$ is a surjection. 
Theorem 3.2. For every $n \in \mathbb{N}$ the canonical map $K_{0}\left(\Omega_{[0,7]}^{n} \mathcal{N}\right) \rightarrow K_{0}\left(\Omega^{n} \mathcal{N}\right) \cong$ $K_{n}(\mathcal{N})$ admits a natural section.

As before, $\mathcal{N}$ denotes an exact category. The basic approach is the same as that of Harris Har15. Section 2.2] in showing that the canonical map from Bass' $K_{1}$ to $K_{0}(\Omega \mathcal{N})$ is an isomorphism for split-exact categories. Our arguments rely on a description of equality of classes in $K_{0}$ of an exact category which is due to Heller [Hel65, Lemma 2.1]. We include a proof following [Tho97, Lemma 2.4] for the reader's convenience.

Definition 3.3. Let $J, K \in \mathcal{N}$.

(1) We call $J$ and $K$ extension-equivalent if there are objects $A, B \in \mathcal{N}$ such that there exist exact sequences

$$
A \longmapsto J \longrightarrow B \text { and } A \succ K \longrightarrow B \text {. }
$$

(2) We call $J$ and $K$ stably extension-equivalent if there exists an object $S \in \mathcal{N}$ such that $J \oplus S$ and $K \oplus S$ are extension-equivalent.

Despite its name, extension-equivalence need not be an equivalence relation. On the other hand, the following lemma shows that stable extension-equivalence is always an equivalence relation.

Lemma 3.4 (Heller). Let $\mathcal{N}$ be an exact category and let $J, J^{\prime}, K, K^{\prime} \in \mathcal{N}$.

Then $[J]-\left[J^{\prime}\right]=[K]-\left[K^{\prime}\right] \in K_{0}(\mathcal{N})$ if and only if $J \oplus K^{\prime}$ and $K \oplus J^{\prime}$ are stably extension-equivalent.

Proof. Define a relation on pairs of objects in $\mathcal{N}$ by setting $\left(J, J^{\prime}\right) \sim\left(K, K^{\prime}\right)$ if and only if $J \oplus K^{\prime}$ and $K \oplus J^{\prime}$ are stably extension-equivalent.

We claim that $\sim$ is an equivalence relation. Reflexivity and symmetry are obvious. To see transitivity, suppose that $\left(J, J^{\prime}\right) \sim\left(K, K^{\prime}\right) \sim\left(L, L^{\prime}\right)$, i.e., there exist $A, B, C, D, S, T \in \mathcal{N}$ such that there are exact sequences

$$
A \longmapsto J \oplus K^{\prime} \oplus S \longrightarrow B \quad \text { and } \quad A \longmapsto K \oplus J^{\prime} \oplus S \longrightarrow B
$$

as well as

$$
C \longmapsto K \oplus L^{\prime} \oplus T \longrightarrow D \quad \text { and } \quad C \longmapsto L \oplus K^{\prime} \oplus T \longrightarrow D .
$$

Then the sequences formed by taking direct sums

$$
\begin{aligned}
& A \oplus C \succ J \oplus K^{\prime} \oplus S \oplus K \oplus L^{\prime} \oplus T \longrightarrow B \oplus D, \\
& A \oplus C \succ K \oplus J^{\prime} \oplus S \oplus L \oplus K^{\prime} \oplus T \longrightarrow B \oplus D,
\end{aligned}
$$

are exact, too. Rewriting

$$
J \oplus K^{\prime} \oplus S \oplus K \oplus L^{\prime} \oplus T \cong J \oplus L^{\prime} \oplus K \oplus K^{\prime} \oplus S \oplus T
$$

and

$$
K \oplus J^{\prime} \oplus S \oplus L \oplus K^{\prime} \oplus T \cong L \oplus J^{\prime} \oplus K \oplus K^{\prime} \oplus S \oplus T
$$

proves transitivity, so $\sim$ is an equivalence relation. Denote by $k(\mathcal{N})$ the set of equivalence classes in $o b \mathcal{N} \times o b \mathcal{N}$ with respect to $\sim$. We write $\left[J, J^{\prime}\right]$ for the class of $\left(J, J^{\prime}\right)$ in $k(\mathcal{N})$. 
Clearly, if $\left(J, J^{\prime}\right)$ and $\left(K, K^{\prime}\right)$ are pairs of objects such that $J \cong K$ and $J^{\prime} \cong K^{\prime}$, then $\left[J, J^{\prime}\right]=\left[K, K^{\prime}\right]$. Hence, the direct sum operation in $\mathcal{N}$ induces the structure of a commutative monoid on $k(\mathcal{N})$ via

$$
\left[J, J^{\prime}\right]+\left[K, K^{\prime}\right]:=\left[J \oplus K, J^{\prime} \oplus K^{\prime}\right] .
$$

It is easy to check that $[J, J]=[0,0]$ for every object $J \in \mathcal{N}$, so $k(\mathcal{N})$ is an abelian group since

$$
\left[J, J^{\prime}\right]+\left[J^{\prime}, J\right]=\left[J \oplus J^{\prime}, J \oplus J^{\prime}\right]=0 .
$$

Let now $J \longmapsto K \rightarrow L$ be an exact sequence in $\mathcal{N}$. Since both

$$
J \longmapsto J \oplus L \longrightarrow L . \quad \text { and } \quad J \longmapsto K \longrightarrow L
$$

are exact, it follows that $[J \oplus L, 0]=[K, 0]$. Hence, the map $o b \mathcal{N} \rightarrow k(\mathcal{N}), J \mapsto[J, 0]$ induces a homomorphism $\varphi: K_{0}(\mathcal{N}) \rightarrow k(\mathcal{N})$.

Note that $\varphi$ sends the class $[J]-\left[J^{\prime}\right] \in K_{0}(\mathcal{N})$ to $\varphi\left([J]-\left[J^{\prime}\right]\right)=\left[J, J^{\prime}\right]$, so $\varphi$ is an epimorphism. Moreover, it is immediate from the definition of $\sim$ that the kernel of $\varphi$ is trivial. This proves that $\varphi$ is an isomorphism, and the claim of the lemma follows.

We can now prove Theorem 3.1 Let $\mathbb{P}:=\left(P_{*}, d, d^{\prime}\right)$ be a binary acyclic complex supported on $[0, m]$ for some $m \in \mathbb{N}$. Choose factorizations $d_{n}: P_{n} \rightarrow J_{n-1} \longmapsto P_{n-1}$ and $d_{n}^{\prime}: P_{n} \rightarrow K_{n-1} \longmapsto P_{n-1}$ for all $n$. Since $J_{n}$ and $K_{n}$ both fit into an exact sequence with $P_{n-1}, \ldots, P_{0}$, they represent the same class in $K_{0}(\mathcal{N})$. Therefore, there exist $A_{n}, B_{n}, S_{n} \in \mathcal{N}$ and exact sequences

$$
A_{n} \succ J_{n} \oplus S_{n} \longrightarrow B_{n} \quad \text { and } \quad A_{n} \succ K_{n} \oplus S_{n} \longrightarrow B_{n} \text {. }
$$

For $n \geq 3$, let $\mathbb{S}_{n}$ denote the binary acyclic complex

$$
A_{n} \rightrightarrows K_{n} \oplus S_{n} \oplus J_{n} \rightrightarrows B_{n} \oplus P_{n} \oplus A_{n-1} \rightrightarrows J_{n-1} \oplus K_{n-1} \oplus S_{n-1} \rightrightarrows B_{n-1}
$$

consisting of top differential

$$
\begin{aligned}
A_{n} \longleftrightarrow K_{n} \underset{\oplus}{\oplus} S_{n} \longrightarrow & B_{n} \\
J_{n} \longmapsto & P_{n} \longrightarrow d_{n} \longrightarrow J_{n-1} \\
P_{\oplus} & A_{n-1} \longrightarrow K_{n-1} \oplus S_{n-1} \longrightarrow B_{n-1}
\end{aligned}
$$

and bottom differential

$$
\begin{aligned}
& A_{n} \longleftrightarrow J_{n} \oplus S_{n} \longrightarrow B_{n} \\
& \stackrel{\oplus}{K_{n}} \longmapsto \stackrel{\oplus}{P_{n}} \longrightarrow d_{n}^{\prime} \longrightarrow K_{n-1} \\
& A_{n-1}^{\oplus} \longleftrightarrow J_{n-1} \stackrel{\oplus}{\oplus} S_{n-1} \longrightarrow B_{n-1} .
\end{aligned}
$$

Note that $\mathbb{S}_{n}$ is zero for almost all $n$. Furthermore, let $\mathbb{S}_{2}$ denote the binary acyclic complex

$$
A_{2} \longrightarrow K_{2} \oplus S_{2} \oplus J_{2} \rightleftarrows B_{2} \oplus P_{2} \rightleftarrows P_{1} \rightleftarrows P_{0}
$$


consisting of top differential

$$
\begin{aligned}
A_{2} & \longrightarrow K_{2} \oplus S_{2} \longrightarrow B_{2} \\
\oplus & \stackrel{\oplus}{\longrightarrow} P_{2} \stackrel{d_{2}}{\longrightarrow} P_{1} \stackrel{d_{1}}{\longrightarrow} P_{0}
\end{aligned}
$$

and bottom differential

$$
\begin{aligned}
A_{2} \longmapsto J_{2} \underset{\oplus}{\oplus} S_{2} & \longrightarrow B_{2} \\
K_{2} & \stackrel{\oplus}{\longrightarrow} P_{2} \stackrel{d_{2}^{\prime}}{\longrightarrow} P_{1} \stackrel{d_{1}^{\prime}}{\longrightarrow} P_{0} .
\end{aligned}
$$

Lemma 3.5. The equation

$$
[\mathbb{P}]=\sum_{n=2}^{\infty}(-1)^{n}\left[\mathbb{S}_{n}\right]
$$

holds in $K_{0}\left(\Omega_{[0, m+3]} \mathcal{N}\right)$.

Proof. Let $\mathbb{P}^{\prime}$ denote the binary acyclic complex

$$
\cdots \Longrightarrow P_{4} \Longrightarrow P_{3} \oplus A_{2} \longrightarrow J_{2} \oplus K_{2} \oplus S_{2} \Longrightarrow B_{2}
$$

with top differential

$$
\begin{aligned}
\ldots \longrightarrow P_{4} \stackrel{d_{4}}{\longrightarrow} P_{3} \stackrel{d_{3}}{\longrightarrow} J_{2} \\
\oplus_{2} \longmapsto K_{2} \oplus S_{2} \longrightarrow B_{2}
\end{aligned}
$$

and bottom differential

$$
\begin{aligned}
\ldots \longrightarrow P_{4} \stackrel{d_{4}^{\prime}}{\longrightarrow} P_{3} \stackrel{d_{3}^{\prime}}{\longrightarrow} K_{2} \\
\stackrel{\oplus}{\oplus} \\
A_{2} \succ J_{2} \oplus S_{2} \longrightarrow B_{2} .
\end{aligned}
$$

We will show that $[\mathbb{P}]=\left[\mathbb{S}_{2}\right]-\left[\mathbb{P}^{\prime}\right]$. The lemma then follows by iterating this procedure. For this notice that the complex $\mathbb{S}_{2}$ for $\mathbb{P}^{\prime}$ is precisely the complex $\mathbb{S}_{3}$ for $\mathbb{P}$. Consider the following binary acyclic double complex. All differentials written as a single arrow are the identity on the summand appearing in domain and codomain and zero on all other summands. In particular, both differentials agree in this case. The remaining four non-trivial binary acyclic complexes are $\mathbb{P}, \mathbb{P}^{\prime}, \mathbb{S}_{2}$, and a fourth one explained in the diagram

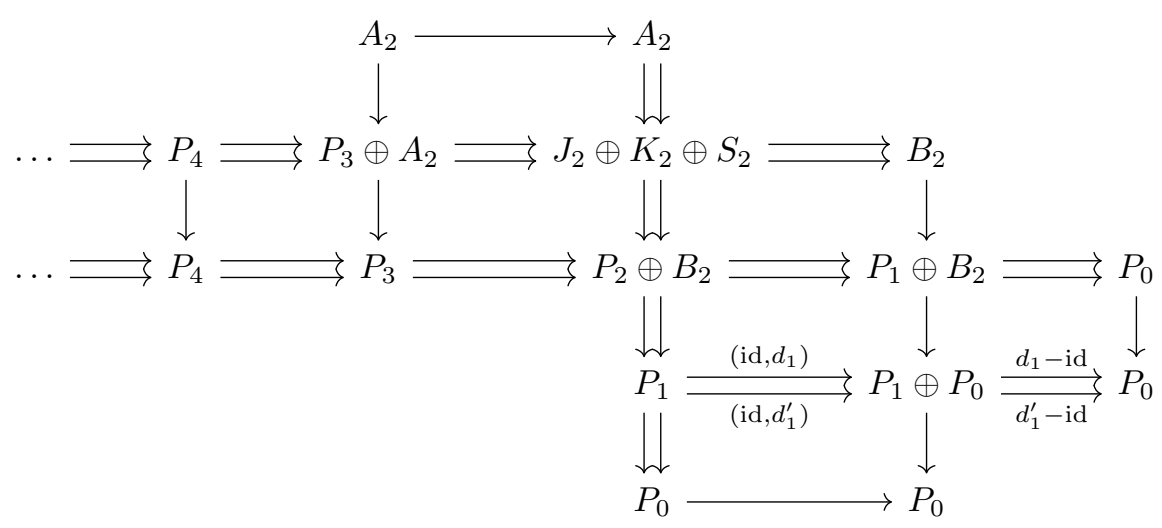


Applying Nenashev's relation (Lemma 2.9) and omitting all summands which are obviously zero, we obtain

$$
-\left[P_{1} \underset{\left(\mathrm{id}, d_{1}^{\prime}\right)}{\stackrel{\left(\mathrm{id}, d_{1}\right)}{\longrightarrow}} P_{1} \oplus P_{0} \underset{d_{1}^{\prime}-\mathrm{id}}{\stackrel{d_{1}-\mathrm{id}}{\longrightarrow}} P_{0}\right]+[\mathbb{P}]-\left[\mathbb{P}^{\prime}[1]\right]=\left[\mathbb{S}_{2}\right] .
$$

We will show that the first summand is trivial. Assuming this, it follows from Lemma 2.7 that

$$
[\mathbb{P}]+\left[\mathbb{P}^{\prime}\right]=\left[\mathbb{S}_{2}\right],
$$

as claimed. In fact, triviality of the binary acyclic complex

$$
P_{1} \underset{\left(\mathrm{id}, d_{1}^{\prime}\right)}{\stackrel{\left(\mathrm{id}, d_{1}\right)}{\longrightarrow}} P_{1} \oplus P_{0} \underset{d_{1}^{\prime}-\mathrm{id}}{\stackrel{d_{1}-\mathrm{id}}{\longrightarrow}} P_{0}
$$

in $K_{0}(\Omega \mathcal{N})$ follows directly from the existence of the following short exact sequence of binary acyclic complexes:

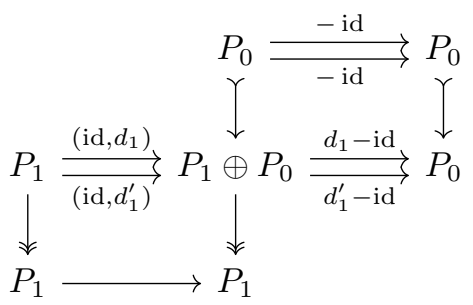

Lemma 3.5 immediately implies Theorem 3.1 since the complexes $\mathbb{S}_{n}$ are supported on $[0,4]$ for all $n \geq 2$.

Our next goal is to prove Theorem 3.2.

Proposition 3.6. The map $K_{0} \Omega \mathcal{N} \rightarrow K_{0} \Omega_{[0,7]} \mathcal{N}$ given by

$$
[\mathbb{P}] \mapsto \sum_{n=2}^{\infty}(-1)^{n}\left[\mathbb{S}_{n}\right]
$$

is a well-defined homomorphism.

Proof. Note that all $J_{n}$ and $K_{n}$ are unique up to isomorphism.

We first show that $\sum_{n=2}^{\infty}(-1)^{n}\left[\mathbb{S}_{n}\right]$ is independent of the choices of $A_{n}, B_{n}, S_{n}$ and the extensions

$$
A_{n} \longleftrightarrow J_{n} \oplus S_{n} \longrightarrow B_{n} \quad \text { and } \quad A_{n} \longleftrightarrow K_{n} \oplus S_{n} \longrightarrow B_{n} .
$$

Fix $k>2$, and let $A_{k}^{\prime}, B_{k}^{\prime}$, and $S_{k}^{\prime}$ be different choices fitting into extensions as $A_{k}$, $B_{k}$, and $S_{k}$. Denote by $\mathbb{S}_{k}^{\prime}$ and $\mathbb{S}_{k+1}^{\prime}$ the same binary acyclic complexes as $\mathbb{S}_{k}$ and $\mathbb{S}_{k+1}$, except that the extensions involving $A_{k}, B_{k}$, and $S_{k}$ are replaced by those involving $A_{k}^{\prime}, B_{k}^{\prime}$, and $S_{k}^{\prime}$. Note that $\mathbb{S}_{l}$ is independent of the choice of $A_{k}, B_{k}$, and $S_{k}$ for $l \neq k, k+1$.

The binary acyclic complexes $\mathbb{S}_{k}^{\prime} \oplus\left(\mathbb{S}_{k+1}[2]\right)$ and $\mathbb{S}_{k} \oplus\left(\mathbb{S}_{k+1}^{\prime}[2]\right)$ have isomorphic underlying graded objects. We regard both as binary acyclic complexes

$$
A_{k+1} \rightrightarrows K_{k+1} \oplus S_{k+1} \oplus J_{k+1} \rightrightarrows B_{k+1} \oplus P_{k+1} \oplus A_{k} \oplus A_{k}^{\prime} \rightrightarrows
$$

$J_{k} \oplus K_{k} \oplus S_{k} \oplus K_{k} \oplus S_{k}^{\prime} \oplus J_{k} \rightrightarrows B_{k} \oplus B_{k}^{\prime} \oplus P_{k} \oplus A_{k-1} \rightrightarrows J_{k-1} \oplus K_{k-1} \oplus S_{k-1} \rightrightarrows B_{k-1}$, 
Both the pair of chain complexes given by the top differentials and the pair of chain complexes given by the bottom differentials of $\mathbb{S}_{k}^{\prime} \oplus\left(\mathbb{S}_{k+1}[2]\right)$ and $\mathbb{S}_{k} \oplus\left(\mathbb{S}_{k+1}^{\prime}[2]\right)$ are isomorphic: The isomorphism for the top differentials has to flip the two copies of $K_{k}$, while the one for the bottom differentials has to flip the two copies of $J_{k}$.

That is, there is the following binary acyclic double complex whose upper row is $\mathbb{S}_{k}^{\prime} \oplus\left(\mathbb{S}_{k+1}[2]\right)$, whose lower row is $\mathbb{S}_{k} \oplus\left(\mathbb{S}_{k+1}^{\prime}[2]\right)$. Here all unmarked downward arrows are the identity, and $\tau_{K}$ and $\tau_{J}$ denote the automorphisms switching the two copies of $K_{k}$ and $J_{k}$, respectively,

$\ldots \rightrightarrows B_{k+1} \oplus P_{k+1} \oplus A_{k} \oplus A_{k}^{\prime} \rightrightarrows J_{k} \oplus K_{k} \oplus S_{k} \oplus K_{k} \oplus S_{k}^{\prime} \oplus J_{k} \rightrightarrows B_{k} \oplus B_{k}^{\prime} \oplus P_{k} \oplus A_{k-1} \rightrightarrows \ldots$

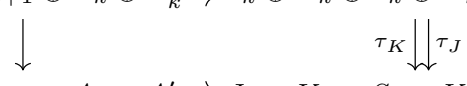

$\ldots \rightrightarrows B_{k+1} \oplus P_{k+1} \oplus A_{k} \oplus A_{k}^{\prime} \rightrightarrows J_{k} \oplus K_{k} \oplus S_{k} \oplus K_{k} \oplus S_{k}^{\prime} \oplus J_{k} \rightrightarrows B_{k} \oplus B_{k}^{\prime} \oplus P_{k} \oplus A_{k-1} \rightrightarrows$...

Applying Lemma 2.9, the difference between the classes of $\mathbb{S}_{k} \oplus\left(\mathbb{S}_{k+1}^{\prime}[2]\right)$ and $\mathbb{S}_{k}^{\prime} \oplus\left(\mathbb{S}_{k+1}[2]\right)$ is therefore the same as

$$
\left[K_{k} \oplus K_{k} \underset{\mathrm{id}}{\stackrel{\tau_{K}}{\longrightarrow}} K_{k} \oplus K_{k}\right]+\left[J_{k} \oplus J_{k} \underset{\tau_{J}}{\stackrel{\mathrm{id}}{\longrightarrow}} J_{k} \oplus J_{k}\right]
$$

in $K_{0}\left(\Omega_{[0,7]} \mathcal{N}\right)$. Since $J_{k}$ and $K_{k}$ represent the same class in $K_{0}$, we have

$$
\left[K_{k} \oplus K_{k} \underset{\text { id }}{\stackrel{\tau_{K}}{\rightrightarrows}} K_{k} \oplus K_{k}\right]=\left[J_{k} \oplus J_{k} \underset{\text { id }}{\stackrel{\tau_{J}}{\rightrightarrows}} J_{k} \oplus J_{k}\right] .
$$

Therefore, $\mathbb{S}_{k}^{\prime} \oplus\left(\mathbb{S}_{k+1}[2]\right)$ and $\mathbb{S}_{k} \oplus\left(\mathbb{S}_{k+1}^{\prime}[2]\right)$ represent the same class in $K_{0}\left(\Omega_{[0,7]} \mathcal{N}\right)$ by Lemma 2.11. In combination with Lemma 2.7, this shows

$$
\left[\mathbb{S}_{k}\right]-\left[\mathbb{S}_{k+1}\right]=\left[\mathbb{S}_{k}^{\prime}\right]-\left[\mathbb{S}_{k+1}^{\prime}\right]
$$

An analogous argument works for $k=2$, so the class $\sum_{n=2}^{\infty}(-1)^{n}\left[\mathbb{S}_{n}\right]$ is independent of the choices we make.

Next, we show that the map is independent of the choice of the representative $\mathbb{P}$ of $[\mathbb{P}]$. First note that if both differentials of the double complex $\mathbb{P}$ agree, then $K_{n}$ and $J_{n}$ agree and we can choose the same extension for both. In this case, both differentials for all $\mathbb{S}_{n}$ agree, so $\left[\mathbb{S}_{n}\right]=0$ for all $n$.

It remains to see that for a short exact sequence $\mathbb{P}^{\prime} \longmapsto \mathbb{P} \rightarrow \mathbb{P}^{\prime \prime}$ we also get short exact sequences $\mathbb{S}_{n}^{\prime} \longmapsto \mathbb{S}_{n} \rightarrow \mathbb{S}_{n}^{\prime \prime}$ for all $n \geq 2$. For every $n$, we have short exact sequences $J_{n}^{\prime} \longmapsto J_{n} \rightarrow J_{n}^{\prime \prime}$ and $K_{n}^{\prime} \longmapsto K_{n} \rightarrow K_{n}^{\prime \prime}$. As above, the $K_{0}$-classes of $K_{n}^{\prime}$ and $J_{n}^{\prime}$ as well as those of $K_{n}^{\prime \prime}$ and $J_{n}^{\prime \prime}$ agree. By the Additivity theorem Qui73, Theorem 2], we have

$$
\left[K_{n}^{\prime} \longmapsto K_{n} \rightarrow K_{n}^{\prime \prime}\right]=\left[J_{n}^{\prime} \longmapsto J_{n} \rightarrow J_{n}^{\prime \prime}\right] \in K_{0}(\mathcal{E N}),
$$

where $\mathcal{E N}$ is the exact category of exact sequences in $\mathcal{N}$. Therefore, we find short exact sequences $A_{n}^{\prime} \longmapsto A_{n} \rightarrow A_{n}^{\prime \prime}, B_{n}^{\prime} \longmapsto B_{n} \rightarrow B_{n}^{\prime \prime}$, and $S_{n}^{\prime} \longmapsto S_{n} \rightarrow S_{n}^{\prime \prime}$ fitting into short exact sequences of short exact sequences:

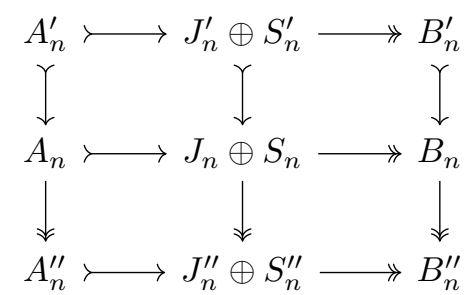

and

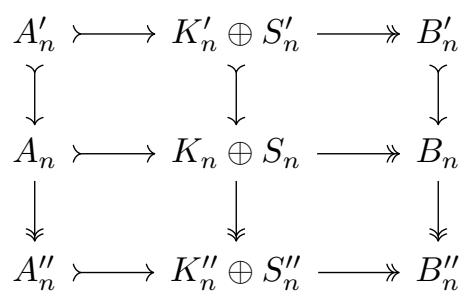


Note that the middle vertical exact sequences are direct sums of the given sequences. Using these extensions for the definition of $\mathbb{S}_{n}^{\prime}, \mathbb{S}_{n}$, and $\mathbb{S}_{n}^{\prime \prime}$, we get the desired short exact sequence $\mathbb{S}_{n}^{\prime} \longmapsto \mathbb{S}_{n} \rightarrow \mathbb{S}_{n}^{\prime \prime}$.

Proof of Theorem 3.2. Lemma 3.5 and Proposition 3.6 prove the case $n=1$. The case $n>1$ follows by induction; compare [Gra12, Remark 8.1].

The map $K_{0}\left(\Omega_{[0,7]}^{n} \mathcal{N}\right) \rightarrow K_{0}\left(\Omega \Omega_{[0,7]}^{n-1} \mathcal{N}\right)$ is a retract of $K_{0}\left(\Omega_{[0,7]}\left(B_{[0,7]}^{q}\right)^{n-1} \mathcal{N}\right) \rightarrow$ $K_{0}\left(\Omega\left(B_{[0,7]}^{q}\right)^{n-1} \mathcal{N}\right)$ which admits a natural section by the case $n=1$. Hence $K_{0}\left(\Omega_{[0,7]}^{n} \mathcal{N}\right) \rightarrow K_{0}\left(\Omega \Omega_{[0,7]}^{n-1} \mathcal{N}\right)$ admits a natural section as well.

Since $\Omega_{[0,7]}$ and $\Omega$ commute, it suffices to show that $K_{0}\left(\Omega_{[0,7]}^{n-1} \Omega \mathcal{N}\right) \rightarrow K_{0}\left(\Omega^{n} \mathcal{N}\right)$ admits a natural section. But this map is a retract of the map $K_{0}\left(\Omega_{[0,7]}^{n-1} B^{q} \mathcal{N}\right) \rightarrow$ $K_{0}\left(\Omega^{n-1} B^{q} \mathcal{N}\right)$, which admits a natural section by the induction assumption.

\section{Algebraic $K$-theory of infinite product CAtegories}

The results of Section 3 allow us to show that the comparison map $\mathbb{K}\left(\prod_{i \in I} \mathcal{N}_{i}\right) \rightarrow$ $\prod_{i \in I} \mathbb{K}\left(\mathcal{N}_{i}\right)$ of connective $K$-theory spectra is a $\pi_{*}$-isomorphism.

Theorem 4.1. For every family $\left\{\mathcal{N}_{i}\right\}_{i \in I}$ of exact categories and every $n \in \mathbb{N}$ the natural map

$$
K_{n}\left(\prod_{i \in I} \mathcal{N}_{i}\right) \rightarrow \prod_{i \in I} K_{n}\left(\mathcal{N}_{i}\right)
$$

is an isomorphism.

Proof. Note that the natural map $K_{0}\left(\prod_{i \in I} \mathcal{N}_{i}\right) \rightarrow \prod_{i \in I} K_{0}\left(\mathcal{N}_{i}\right)$ is clearly surjective, and that injectivity is a consequence of Lemma 3.4

Recall that $K_{n}(\mathcal{N})$ is naturally isomorphic to $K_{0}\left(\Omega^{n} \mathcal{N}\right)$. Consider the following diagram, where the vertical maps are the sections from Theorem 3.2 followed by the canonical homomorphisms:

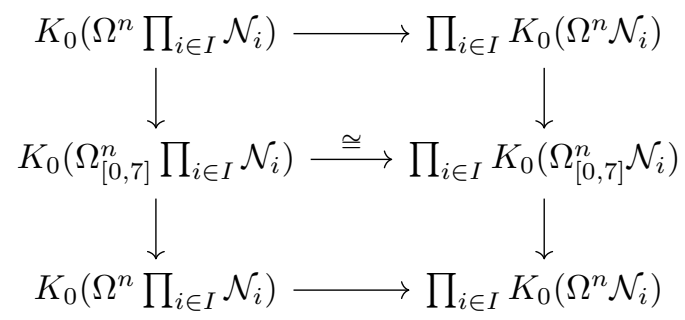

Since the natural functors $C_{[0,7]}^{q}\left(\prod_{i \in I} \mathcal{N}_{i}\right) \rightarrow \prod_{i \in I} C_{[0,7]}^{q}\left(\mathcal{N}_{i}\right)$ and $B_{[0,7]}^{q}\left(\prod_{i \in I} \mathcal{N}_{i}\right) \rightarrow$ $\prod_{i \in I} B_{[0,7]}^{q}\left(\mathcal{N}_{i}\right)$ are isomorphisms, the middle horizontal map is an isomorphism. A diagram chase implies that the natural map $K_{n}\left(\prod_{i \in I} \mathcal{N}_{i}\right) \rightarrow \prod_{i \in I} K_{n}\left(\mathcal{N}_{i}\right)$ is an isomorphism.

In the remainder of this section, we extend this statement to non-connective $K$-theory. Our model for the non-connective algebraic $K$-theory $\mathbb{K}^{-\infty}$ of an exact category is Schlichting's delooping [Sch06, Section 12].

The argument to extend Theorem 4.1 to non-connective algebraic $K$-theory is based on a localization sequence of Schlichting Sch04]. To state it, we need to recall the following definition. 
Definition 4.2 ([Sch04, Definitions 1.3 and 1.5]). Let $\mathcal{N}$ be an exact category, and let $\mathcal{A} \subseteq \mathcal{N}$ be an extension closed full subcategory.

(1) An admissible epimorphism $N \rightarrow A$ with $N \in \mathcal{N}$ and $A \in \mathcal{A}$ is special if there exists an admissible monomorphism $B \longmapsto N$ with $B \in \mathcal{A}$ such that the composition $B \rightarrow A$ is an admissible epimorphism.

(2) The inclusion $\mathcal{A} \subseteq \mathcal{N}$ is called left s-filtering if the following holds:

(a) The subcategory $\mathcal{A}$ is closed under admissible subobjects and admissible quotients in $\mathcal{N}$.

(b) Every admissible epimorphism $N \rightarrow A$ from an object $N \in \mathcal{N}$ to an object $A \in \mathcal{A}$ is special.

(c) For every morphism $f: A \rightarrow N$ with $A \in \mathcal{A}$ and $N \in \mathcal{N}$ there exists an object $B \in \mathcal{A}$, a morphism $f^{\prime}: A \rightarrow B$, and an admissible monomorphism $i: B \longmapsto N$ such that $i f^{\prime}=f$.

Let $\mathcal{A} \subseteq \mathcal{N}$ be a left $s$-filtering subcategory. A weak isomorphism in $\mathcal{N}$ is a morphism which can be written as the composition of admissible monomorphisms with cokernel in $\mathcal{A}$ and admissible epimorphisms with kernel in $\mathcal{A}$. Let $\Sigma$ denote the collection of weak isomorphisms in $\mathcal{N}$. The set $\Sigma$ satisfies a calculus of left fractions [Sch04, Lemma 1.13], so one can form the localization $\mathcal{N}\left[\Sigma^{-1}\right]$. The localization inherits an exact structure from $\mathcal{N}$ by declaring a sequence to be exact if it is isomorphic to the image of an exact sequence under the localization functor $\mathcal{N} \rightarrow \mathcal{N}\left[\Sigma^{-1}\right]$ [Sch04, Proposition 1.16]. The resulting exact category is denoted $\mathcal{N} / \mathcal{A}$

Theorem 4.3 ([Sch04, Theorem 2.10]). Let $\mathcal{A}$ be an idempotent complete, left $s$ filtering subcategory of the exact category $\mathcal{N}$. Then the sequence $\mathcal{A} \rightarrow \mathcal{N} \rightarrow \mathcal{N} / \mathcal{A}$ of exact functors induces a homotopy fiber sequence of spectra

$$
\mathbb{K}^{-\infty}(\mathcal{A}) \rightarrow \mathbb{K}^{-\infty}(\mathcal{N}) \rightarrow \mathbb{K}^{-\infty}(\mathcal{N} / \mathcal{A})
$$

Finally, recall the countable envelope $\mathcal{F N}$ of an idempotent complete exact category $\mathcal{N}$ [Sch04, Section 3] (and the references therein). The concrete definition need not concern us here. It suffices to know that $\mathcal{F N}$ is an exact category which contains $\mathcal{N}$ as a left $s$-filtering subcategory, and that $\mathbb{K}^{-\infty}(\mathcal{F} \mathcal{N})$ is contractible [Sch04, Lemma 3.2]; the latter claim holds because $\mathcal{F N}$ admits countable coproducts. Moreover, $\mathcal{F} \mathcal{N}$ depends functorially on $\mathcal{N}$. Denote by $\mathcal{S} \mathcal{N}$ the quotient category $\mathcal{F} \mathcal{N} / \mathcal{N}$. The category $\mathcal{S N}$ is called the suspension of $\mathcal{N}$. Write $\mathcal{S}^{n} \mathcal{N}$ for the $n$-fold suspension of $\mathcal{N}$. From Theorem 4.3, it follows directly that $\Omega^{n} \mathbb{K}^{-\infty}\left(\mathcal{S}^{n} \mathcal{N}\right)$ is naturally equivalent to $\mathbb{K}^{-\infty}(\mathcal{N})$. In particular, we have $K_{-n}(\mathcal{N}) \cong K_{0}\left(\operatorname{Idem}\left(\mathcal{S}^{n} \mathcal{N}\right)\right)$ for all $n>0$, where $\operatorname{Idem}(-)$ denotes the idempotent completion functor.

Proof of Theorem 1.2, Let $\left\{\mathcal{N}_{i}\right\}_{i \in I}$ be a family of exact categories.

Since the natural map $\mathbb{K}^{-\infty}(\mathcal{N}) \stackrel{\sim}{\longrightarrow} \mathbb{K}^{-\infty}(\operatorname{Idem}(\mathcal{N}))$ is an equivalence for every exact category and $\operatorname{Idem}\left(\prod_{i \in I} \mathcal{N}_{i}\right) \cong \prod_{i \in I} \operatorname{Idem}\left(\mathcal{N}_{i}\right)$, we may assume that $\mathcal{N}_{i}$ is idempotent complete for all $i \in I$.

Consider the left $s$-filtering inclusion $\prod_{i \in I} \mathcal{N}_{i} \subseteq \mathcal{F}\left(\prod_{i \in I} \mathcal{N}_{i}\right)$. The various projection functors $\prod_{i \in I} \mathcal{N}_{i} \rightarrow \mathcal{N}_{j}$ induce an exact functor $\mathcal{F}\left(\prod_{i \in I} \mathcal{N}_{i}\right) \rightarrow \prod_{i \in I} \mathcal{F} \mathcal{N}_{i}$. Moreover, the inclusion $\prod_{i \in I} \mathcal{N}_{i} \subset \prod_{i \in I} \mathcal{F} \mathcal{N}_{i}$ is left $s$-filtering since it is left $s$ filtering on each factor. Since $\mathcal{S N}_{i}$ is obtained from $\mathcal{F} \mathcal{N}_{i}$ by a calculus of left 
fractions, we can identify $\prod_{i \in I} \mathcal{F} \mathcal{N}_{i} / \prod_{i \in I} \mathcal{N}_{i} \cong \prod_{i \in I} \mathcal{S N}_{i}$ (for an explicit description of the morphism sets making this obvious, cf. [GZ67, Chapter I, Section 2.2]). Therefore, we have by Theorem 4.3 a map of homotopy fiber sequences of spectra:

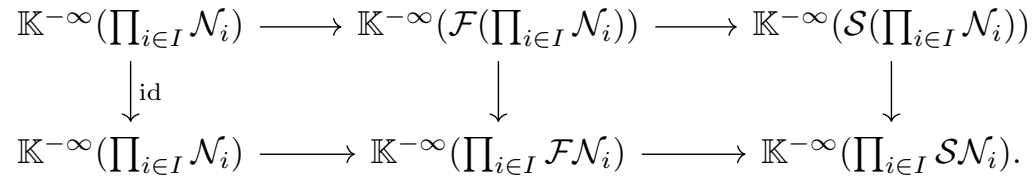

Since both $\mathcal{F}\left(\prod_{i \in I} \mathcal{N}_{i}\right)$ and $\prod_{i \in I} \mathcal{F} \mathcal{N}_{i}$ admit countable coproducts, the $K$-theory of both vanishes and the middle vertical arrow is a $\pi_{*}$-isomorphism. Hence, the right vertical map is a $\pi_{*}$-isomorphism. By induction, it follows that the canonical map

$$
\mathbb{K}^{-\infty}\left(\mathcal{S}^{n}\left(\prod_{i \in I} \mathcal{N}_{i}\right)\right) \rightarrow \mathbb{K}^{-\infty}\left(\prod_{i \in I} \mathcal{S}^{n} \mathcal{N}_{i}\right)
$$

is a $\pi_{*}$-isomorphism for every family of idempotent complete exact categories.

Let $n>0$. We have the commutative diagram

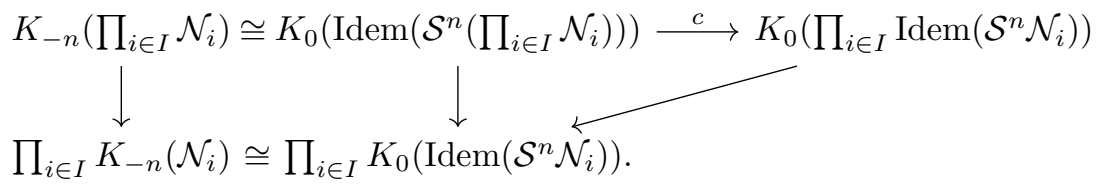

The map $c$ is an isomorphism as we have just discussed. Since the diagonal map is an isomorphism by Theorem 4.1, the theorem follows.

Remark 4.4. Note that the proof for negative $K$-groups only used that $K_{0}$ commutes with infinite products, which was a direct consequence of Lemma 3.4.

\section{The Relation to Nenashev's $K_{1}$}

The abelian group $K_{0}(\Omega \mathcal{N})$ is not the first algebraic description of $K_{1}$ of an exact category. Nenashev gave the following description of $K_{1}(\mathcal{N})$.

Definition 5.1. Define $K_{1}^{N}(\mathcal{N})$ as the abelian group generated by binary acyclic complexes of length two

$$
\mathbb{P}=P_{2} \rightleftarrows P_{1} \Longrightarrow P_{0}
$$

subject to the following relations:

(1) If the top and bottom differential of a binary acyclic complex coincide, that complex represents zero.

(2) For any binary acyclic double complex (see Remark 2.10)

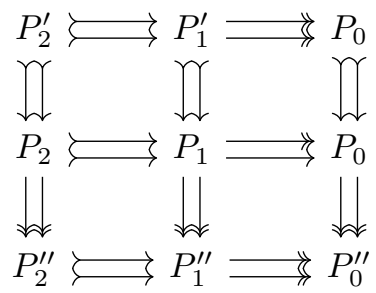

we have

$$
\left[\mathbb{P}_{0}\right]-\left[\mathbb{P}_{1}\right]+\left[\mathbb{P}_{2}\right]=\left[\mathbb{P}^{\prime}\right]-[\mathbb{P}]+\left[\mathbb{P}^{\prime \prime}\right]
$$


The main result of Nen98 states that $K_{1}^{N}(\mathcal{N})$ is isomorphic to $K_{1}(\mathcal{N})$. By Lemma 2.9. regarding a binary acyclic complex of length two as a class in $K_{0}(\Omega \mathcal{N})$ defines a natural homomorphism

$$
\Phi: K_{1}^{N}(\mathcal{N}) \rightarrow K_{0}(\Omega \mathcal{N}),
$$

as already remarked in the introduction. In this section, we prove Theorem 1.1. Before doing so, we give the following corollary.

Corollary 5.2. For all $n \geq 1$, the homomorphism $K_{0}\left(\Omega_{[0,2]}^{n} \mathcal{N}\right) \rightarrow K_{0}\left(\Omega^{n} \mathcal{N}\right)$ is a surjection and the homomorphism $K_{0}\left(\Omega_{[0,4]}^{n} \mathcal{N}\right) \rightarrow K_{0}\left(\Omega^{n} \mathcal{N}\right)$ admits a natural section.

Proof. By Theorem 1.1, $\Phi$ is an isomorphism. Since $K_{0}\left(\Omega_{[0,2]} \mathcal{N}\right) \rightarrow K_{1}^{N}(\mathcal{N})$ is a surjection, so is $K_{0}\left(\Omega_{[0,2]} \mathcal{N}\right) \rightarrow K_{0}(\Omega \mathcal{N})$. By Lemma 2.9. $\Phi$ factors as

$$
\Phi: K_{1}^{N}(\mathcal{N}) \rightarrow K_{0}\left(\Omega_{[0,4]} \mathcal{N}\right) \rightarrow K_{0}(\Omega \mathcal{N}) .
$$

This exhibits $K_{0}(\Omega \mathcal{N})$ as a natural retract of $K_{0}\left(\Omega_{[0,4]} \mathcal{N}\right)$. For $n>1$, the claim follows as in the proof of Theorem 3.2 by induction.

Hence, Theorem 1.1 also proves that the algebraic $K$-theory functor commutes with infinite products.

In the remainder of this section, we give a proof of Theorem 1.1 As in the proof of Theorem 3.2. this will be accomplished by producing an explicit formula that expresses the class of an arbitrary binary acyclic complex in terms of binary acyclic complexes of length two.

Before we start shortening binary acyclic complexes, we make a quick observation about $K_{1}^{N}(\mathcal{N})$, which we will need later in the argument.

Lemma 5.3. For any binary acyclic complex of length two, we have

$$
\left[P_{2} \underset{d_{2}^{\prime}}{\stackrel{d_{2}}{\longrightarrow}} P_{1} \underset{d_{1}^{\prime}}{\stackrel{d_{1}}{\rightleftarrows}} P_{0}\right]=-\left[P_{2} \underset{d_{2}}{\stackrel{d_{2}^{\prime}}{\rightleftarrows}} P_{1} \underset{d_{1}}{\stackrel{d_{1}^{\prime}}{\rightrightarrows}} P_{0}\right] \in K_{1}^{N}(\mathcal{N}) \text {. }
$$

Proof. This follows directly from applying the defining relations of $K_{1}^{N}$ to the binary acyclic double complex

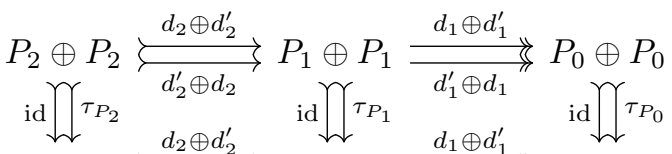

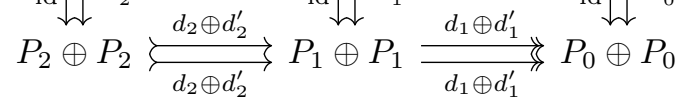

Let $\mathbb{P}:=\left(P_{*}, d, d^{\prime}\right)$ be a binary acyclic complex. In a first step we will not shorten $\mathbb{P}$ but produce a complex $\widehat{\mathbb{P}}$ representing the same class in $K_{0}(\Omega \mathcal{N})$, which we will then be able to shorten.

Choose factorizations $d_{2}: P_{2} \rightarrow J \longmapsto P_{1}$ and $d_{2}^{\prime}: P_{2} \rightarrow K \longmapsto P_{1}$. Since $J$ and $K$ both are the kernel of an admissible epimorphism $P_{1} \rightarrow P_{0}$, they represent the same class in $K_{0}(\mathcal{N})$. Therefore, there exist by Lemma 3.4 $A, B, S \in \mathcal{N}$, and exact sequences

$$
A \longmapsto J \oplus S \longrightarrow B \text { and } A \succ K \oplus S \longrightarrow B .
$$


Let $\mathbb{S}$ denote the binary acyclic complex

$$
A \longrightarrow K \oplus S \oplus J \longrightarrow B \oplus P_{1} \longrightarrow P_{0}
$$

consisting of top differential

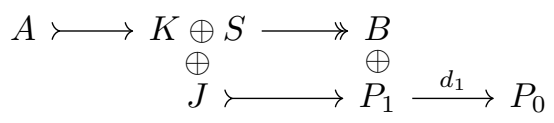

and bottom differential

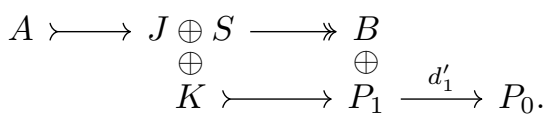

Let $\mathbb{P}^{\prime}$ denote the binary acyclic complex

$$
\ldots \rightleftarrows P_{3} \rightleftarrows P_{2} \oplus A \longrightarrow J \oplus K \oplus S \rightrightarrows B
$$

with top differential

$$
\begin{aligned}
& \ldots \longrightarrow P_{3} \stackrel{d_{3}}{\longrightarrow} P_{2} \stackrel{d_{2}}{\longrightarrow} \underset{\oplus}{J} \\
& \oplus_{A} \longmapsto K \oplus S \longrightarrow B
\end{aligned}
$$

and bottom differential

$$
\begin{aligned}
\ldots \longrightarrow P_{3} \stackrel{d_{3}^{\prime}}{\longrightarrow}{\underset{P}{2}}_{\oplus} \stackrel{d_{2}^{\prime}}{\longrightarrow} \underset{\oplus}{K} \\
A \longmapsto J \oplus S \longrightarrow B .
\end{aligned}
$$

For an object $M \in \mathcal{N}$ we denote by $\Delta_{M}$ the binary acyclic complex

$$
M \underset{\operatorname{id}_{M}}{\stackrel{\mathrm{id}_{M}}{\longrightarrow}} M .
$$

Note that $\left[\Delta_{M}\right]=0 \in K_{0}(\Omega \mathcal{N})$.

Consider the following binary acyclic double complex. All differentials written as a single arrow are the identity on the summand appearing in domain and codomain and zero on all other summands. In particular, both differentials agree in this case. The remaining four non-trivial binary acyclic complexes are $\mathbb{P} \oplus \Delta_{B}, \mathbb{P}^{\prime}$, and $\mathbb{S}$

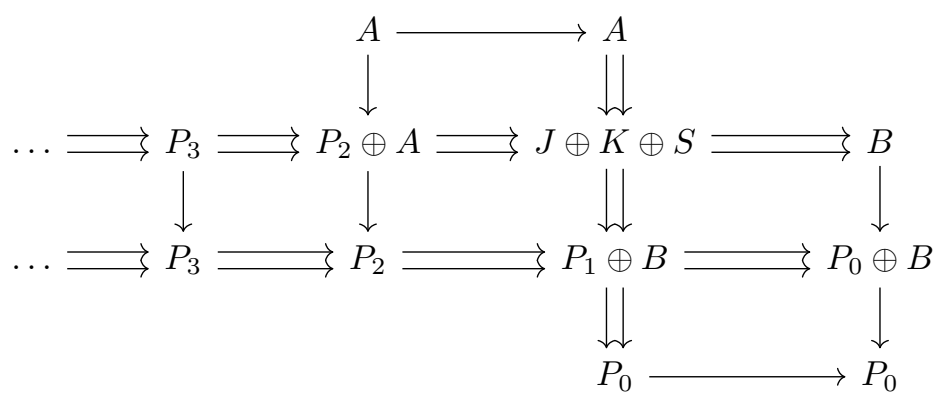

Applying Nenashev's relation (Lemma 2.9) and omitting all summands which are obviously zero, we obtain

$$
[\mathbb{P}]-\left[\mathbb{P}^{\prime}\right]=[\mathbb{S}]
$$


Let $\widehat{\mathbb{P}}$ denote the binary acyclic complex

$$
\ldots \Longrightarrow P_{3} \Longrightarrow P_{2} \oplus J \oplus K \Longrightarrow J \oplus P_{1} \oplus K \Longrightarrow P_{0}
$$

with top differential

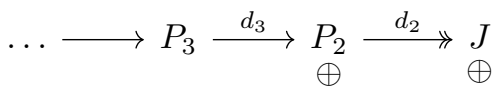

$$
\begin{aligned}
& \stackrel{\oplus}{J} \longleftrightarrow \stackrel{\oplus}{P_{1}} \stackrel{d_{1}}{\longrightarrow} P_{0} \\
& \stackrel{\oplus}{\longrightarrow} \stackrel{\operatorname{id}_{K}}{\longrightarrow} \stackrel{\oplus}{K}
\end{aligned}
$$

and bottom differential

$$
\begin{aligned}
& \ldots \longrightarrow P_{3} \stackrel{d_{3}^{\prime}}{\longrightarrow} P_{2} \stackrel{d_{2}^{\prime}}{\longrightarrow} K \\
& \stackrel{\oplus}{K} \longleftrightarrow \stackrel{\oplus}{P_{1}} \stackrel{d_{1}^{\prime}}{\longrightarrow} P_{0}
\end{aligned}
$$

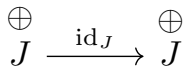

We can build the following binary acyclic double complex involving $\widehat{\mathbb{P}} \oplus \Delta_{B}, \mathbb{P}^{\prime} \oplus$ $\Delta_{J}[1] \oplus \Delta_{K}[1]$, and a third non-trivial complex.

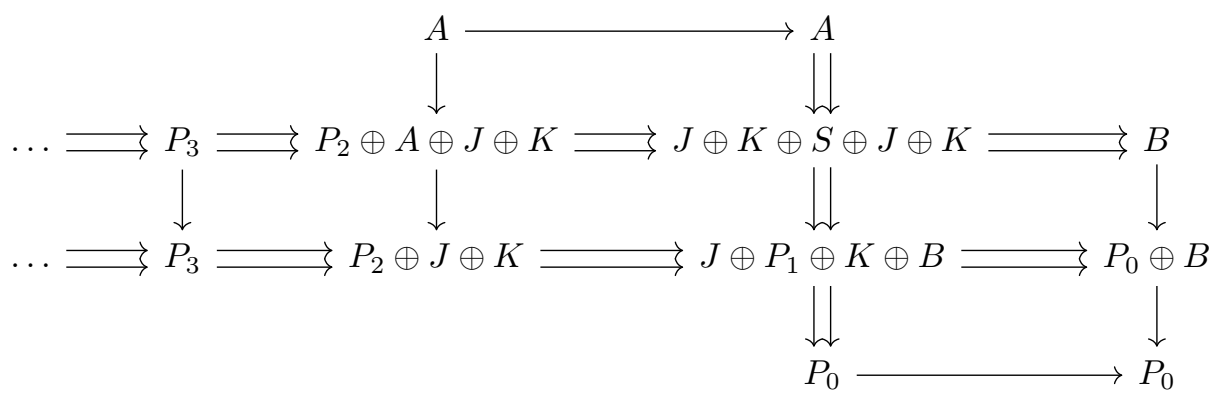

The non-trivial vertical complex differs from $\Delta_{J}[1] \oplus \mathbb{S} \oplus \Delta_{K}[1]$ by identifying $J \oplus P_{1} \oplus K \oplus B \cong J \oplus B \oplus P_{1} \oplus K$ and using $\tau_{K}$ and $\tau_{J}$ in the bottom differential. Since $K$ and $J$ represent the same class in $K_{0}$, we have

$$
\left[K \oplus K \underset{\tau_{K}}{\stackrel{\mathrm{id}}{\longrightarrow}} K \oplus K\right]=\left[J \oplus J \underset{\tau_{J}}{\stackrel{\mathrm{id}}{\longrightarrow}} J \oplus J\right]
$$

and thus the vertical complex represents the same class as $\mathbb{S}$ by Lemma 2.11.

Applying Nenashev's relation (Lemma 2.9) and omitting all summands which are obviously zero, we obtain

$$
[\widehat{\mathbb{P}}]-\left[\mathbb{P}^{\prime}\right]=[\mathbb{S}]
$$

and thus

$$
[\widehat{\mathbb{P}}]=[\mathbb{P}] .
$$

Let $\mathbb{Q}$ denote the binary acyclic complex

$$
\ldots \rightleftarrows P_{4} \rightleftarrows P_{3} \oplus K \oplus J \longrightarrow P_{2} \oplus P_{1} \oplus J \oplus K \rightrightarrows J \oplus P_{0} \oplus K
$$


with top differential

$$
\begin{aligned}
& \ldots \longrightarrow P_{4} \stackrel{d_{4}}{\longrightarrow} P_{3} \stackrel{d_{3}}{\longrightarrow} P_{2} \stackrel{d_{2}}{\longrightarrow J} \\
& \stackrel{\oplus}{K} \longmapsto \stackrel{\oplus}{P_{1}} \stackrel{d_{1}^{\prime}}{\longrightarrow} \stackrel{\oplus}{P_{0}}
\end{aligned}
$$

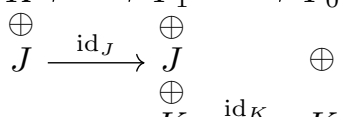

$$
\begin{aligned}
& \stackrel{\oplus}{\mathrm{in}} \stackrel{\mathrm{id}_{K}}{\longrightarrow} K
\end{aligned}
$$

and bottom differential

$$
\begin{aligned}
& \ldots \longrightarrow P_{4} \stackrel{d_{4}^{\prime}}{\longrightarrow} P_{3} \stackrel{d_{3}^{\prime}}{\longrightarrow} P_{2} \stackrel{d_{2}^{\prime}}{\longrightarrow} K \\
& \stackrel{\oplus}{J} \longleftrightarrow \stackrel{\oplus}{P_{1}} \stackrel{d_{1}}{\longrightarrow} \stackrel{\oplus}{P_{0}}
\end{aligned}
$$

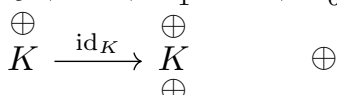

$$
\begin{aligned}
& \stackrel{\oplus}{\stackrel{\mathrm{id}_{J}}{\longrightarrow} J}
\end{aligned}
$$

Let us fix the following notation: If $M$ is an object containing $N$ as a direct summand, denote by $e_{N}$ the obvious idempotent $M \rightarrow M$ whose image is $N$.

Consider the following double complex involving $\widehat{\mathbb{P}}$ and $\mathbb{Q}[1]$. Note that only the rows are acyclic. This suffices to see that the total complex shifted down by one represents the same class as $[\mathbb{Q}]+[\widehat{\mathbb{P}}]$

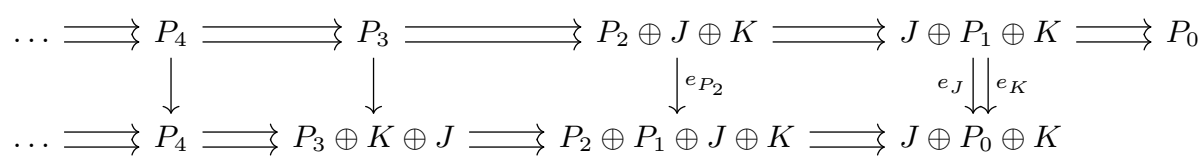

Let $\mathbb{T}$ denote the total complex shifted down by one. Then $\mathbb{T}$ is

$$
\ldots \Longrightarrow P_{4} \oplus P_{3} \Longrightarrow \begin{gathered}
P_{3} \oplus K \oplus J \oplus \\
P_{2} \oplus J \oplus K
\end{gathered} \Longrightarrow \begin{gathered}
P_{2} \oplus P_{1} \oplus J \oplus \\
K \oplus J \oplus P_{1} \oplus K
\end{gathered} \Longrightarrow J \oplus P_{0} \oplus K \oplus P_{0}
$$

Assume that $\mathbb{P}$ was supported on $[0, m]$, then $\mathbb{T}$ admits a projection onto $\Delta_{P_{m}}[m-1]$. The kernel of this projection admits a projection to $\Delta_{P_{m-1}}[m-2]$ and so on until we take the kernel of the projection to $\Delta_{P_{2}}[1]$. The remaining acyclic binary complex $\mathbb{T}^{\prime}$ is

$$
K \oplus J \oplus J \oplus K \longrightarrow P_{1} \oplus J \oplus K \oplus J \oplus P_{1} \oplus K \longrightarrow J \oplus P_{0} \oplus K \oplus P_{0}
$$

with top differential

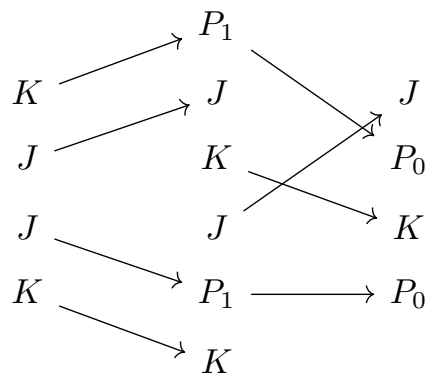


and bottom differential

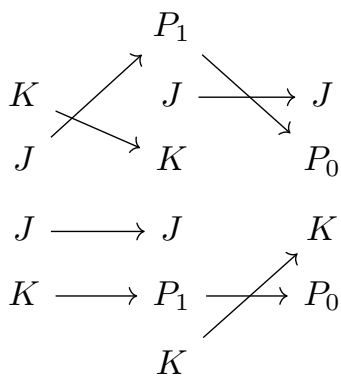

It follows that $[\mathbb{P}]=\left[\mathbb{T}^{\prime}\right]-[\mathbb{Q}]$. Since $\mathbb{T}^{\prime}$ is supported on $[0,2]$ and $\mathbb{Q}$ has length one shorter than $\mathbb{P}$, iterating this argument already shows that $K_{0}\left(\Omega_{[0,2]} \mathcal{N}\right) \rightarrow K_{0}(\Omega \mathcal{N})$ is surjective.

Remark 5.5. The idea to use the complexes $\widehat{\mathbb{P}}, \mathbb{T}$, and $\mathbb{T}^{\prime}$ is from the aforementioned, unpublished result of Grayson. He uses a different argument to show that $[\widehat{\mathbb{P}}]-[\mathbb{P}]$ is contained in the image of $\Phi$. Grayson's argument avoids the use of Heller's lemma, but the computation of the appearing correction terms is more complicated.

We now want to simplify $\mathbb{T}^{\prime}$. Let $\mathbb{T}_{\text {triv }}^{\prime}$ denote the binary acyclic complex whose underlying graded object is that of $\mathbb{T}^{\prime}$, but with both differentials equal to the top differential of $\mathbb{T}^{\prime}$. Then the following diagram, where the upper row is $\mathbb{T}^{\prime}$ and the second row is $\mathbb{T}_{\text {triv }}^{\prime}$, commutes:

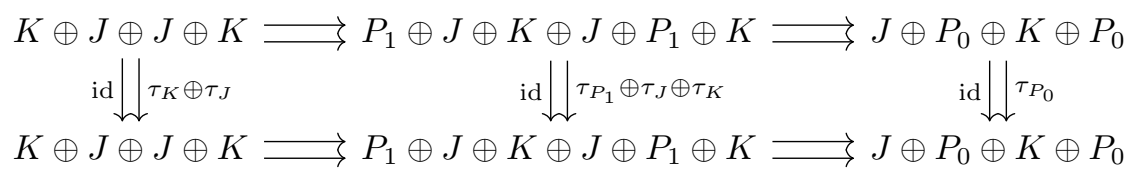

Both differentials of $\mathbb{T}_{\text {triv }}^{\prime}$ agree and thus it represents the trivial class. Since $\tau_{K}$ and $\tau_{J}$ are of order two, we conclude from Lemma 2.9 that

$$
\left[\mathbb{T}^{\prime}\right]=\left[P_{0} \oplus P_{0} \underset{\tau_{P_{0}}}{\stackrel{\mathrm{id}}{\longrightarrow}} P_{0} \oplus P_{0}\right]-\left[P_{1} \oplus P_{1} \underset{\tau_{P_{1}}}{\stackrel{\mathrm{id}}{\longrightarrow}} P_{1} \oplus P_{1}\right] .
$$

Since $J \longmapsto P_{1} \rightarrow P_{0}$ is exact, this is the same as $\left[J \oplus J \underset{\tau_{J}}{\stackrel{\text { id }}{\rightrightarrows}} J \oplus J\right]$. This shows that

$$
[\mathbb{P}]=\left[J \oplus J \underset{\tau_{J}}{\stackrel{\mathrm{id}}{\rightrightarrows}} J \oplus J\right]-[\mathbb{Q}] .
$$

We are now going to iterate this argument. Choose factorizations $d_{n}: P_{n} \rightarrow$ $J_{n-1} \longmapsto P_{n-1}$ and $d_{n}^{\prime}: P_{n} \rightarrow K_{n-1} \longmapsto P_{n-1}$ for all $n \geq 2$ such that $J_{n} \longmapsto P_{n} \rightarrow$ $J_{n-1}$ and $K_{n} \longmapsto P_{n} \rightarrow K_{n-1}$ are exact for all $n$. Set $J_{0}:=P_{0}$ and $K_{0}:=P_{0}$. For 
any natural number $k$, fix the following auxiliary notation:

$$
\begin{array}{rlrl}
J_{\text {odd }}^{k} & :=\bigoplus_{\substack{n \leq k, n \text { odd }}} J_{n}, & J_{\mathrm{ev}}^{k}:=\bigoplus_{\substack{2 \leq n \leq k, n \text { even }}} J_{n}, & J_{\mathrm{ev}, 0}^{k}:=\bigoplus_{\substack{n \leq k, n \text { even }}} J_{n}, \\
K_{\text {odd }}^{k}:=\bigoplus_{\substack{n \leq k, n \text { odd }}}^{k} K_{n}, & K_{\mathrm{ev}}^{k}:=\bigoplus_{\substack{2 \leq n \leq k, n \text { even }}} K_{n}, \quad K_{\mathrm{ev}, 0}^{k}:=\bigoplus_{\substack{n \leq k, n \text { even }}} K_{n}, \\
P_{\text {odd }}^{k}:=\bigoplus_{\substack{n \leq k, n \text { odd }}} P_{n}, & P_{\mathrm{ev}}^{k}:=\bigoplus_{\substack{2 \leq n \leq k \\
n \text { even }}} P_{n} .
\end{array}
$$

First of all, we define for every natural number $k$ a binary acyclic complex $\mathbb{P}_{k}$ of the form

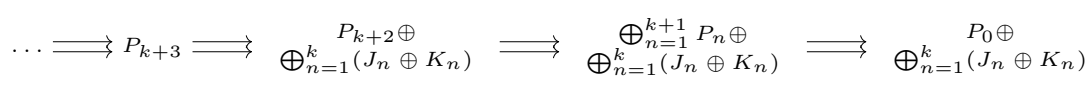

For even natural numbers $k$, we equip $\mathbb{P}_{k}$ with the top differential

$$
\begin{aligned}
& \ldots \longrightarrow P_{k+3} \stackrel{d_{k+3}}{\longrightarrow} P_{k+2} \stackrel{d_{k+2}}{\longrightarrow} P_{k+1} \stackrel{d_{k+1}}{\longrightarrow} J_{k} \\
& \stackrel{\oplus}{K_{\text {odd }}^{k}} \longrightarrow K_{\text {odd }}^{\stackrel{\oplus}{*}} \\
& \oplus \oplus \oplus \\
& J_{\mathrm{ev}}^{k} \longrightarrow J_{\mathrm{ev}}^{k} \\
& K_{\mathrm{ev}}^{k} \longrightarrow K_{\mathrm{ev}}^{k} \\
& \oplus \quad \stackrel{\oplus}{J_{\text {odd }}^{k}} \longrightarrow J_{\text {odd }}^{k} \\
& J_{\text {odd }}^{k} \longrightarrow P_{\text {odd }}^{\oplus} \longrightarrow J_{\mathrm{ev}, 0}^{k-1} \\
& \oplus \oplus \oplus \\
& K_{\mathrm{ev}}^{k} \longrightarrow P_{\mathrm{ev}}^{k} \longrightarrow K_{\mathrm{odd}}^{k}
\end{aligned}
$$

and bottom differential

$$
\begin{aligned}
& \ldots \longrightarrow P_{k+3} \stackrel{d_{k+3}^{\prime}}{\longrightarrow} P_{k+2} \stackrel{d_{k+2}^{\prime}}{\longrightarrow} P_{k+1} \stackrel{d_{k+1}^{\prime}}{\longrightarrow} K_{k} \\
& \underset{\oplus}{\oplus} J_{\text {odd }}^{k} \longrightarrow \underset{\text { odd }}{J^{k}} \\
& K_{\mathrm{ev}}^{k} \longrightarrow K_{\mathrm{ev}}^{k} \\
& J_{\mathrm{ev}}^{\oplus} \longrightarrow J_{\mathrm{ev}}^{k} \\
& \oplus \\
& \stackrel{\oplus}{K_{\text {odd }}^{k}} \longrightarrow K_{\text {odd }}^{k} \\
& K_{\mathrm{odd}}^{k} \longrightarrow P_{\mathrm{odd}}^{k} \longrightarrow K_{\mathrm{ev}, 0}^{k-1} \\
& J_{\mathrm{ev}}^{\oplus} \longrightarrow \stackrel{\oplus}{\oplus} P_{\mathrm{ev}}^{k} \longrightarrow J_{\mathrm{odd}}^{k}
\end{aligned}
$$

Note that $\mathbb{P}_{0}$ is precisely the complex $\mathbb{P}$. 
For odd natural numbers $k$, we equip $\mathbb{P}_{k}$ with the top differential

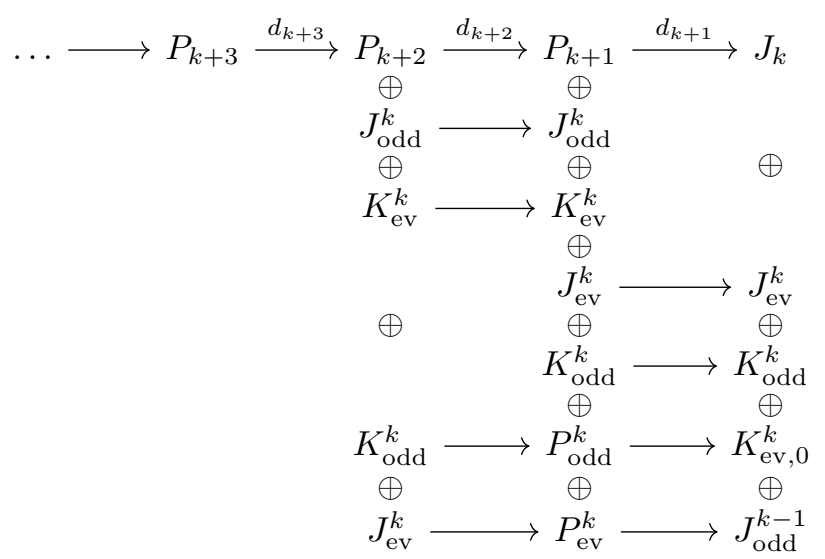

and bottom differential

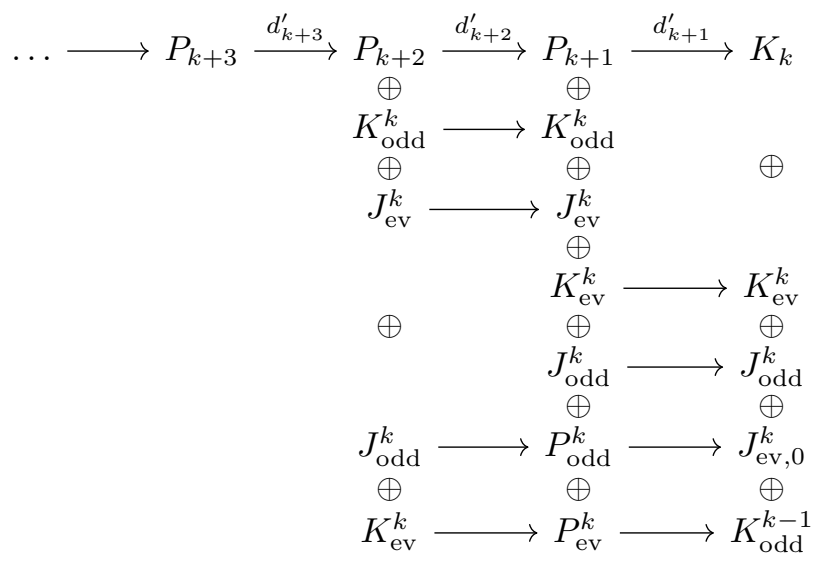

Note that $\mathbb{P}_{1}$ is precisely the complex $\mathbb{Q}$ appearing in (5.6). Moreover, if $k$ is sufficiently large so that $P_{n} \cong 0$ for all $n>k$, then $\mathbb{P}_{k+1}$ is obtained from $\mathbb{P}_{k}$ by interchanging the top and bottom differential.

For every $k$ let $\mathbb{Q}_{k}$ denote the complex obtained from $\mathbb{P}_{k}$ by the same procedure as $\mathbb{Q}$ is obtained from $\mathbb{P}$.

Suppose now that $k$ is odd. Substituting appropriately in (5.6), we obtain the equation

$$
\left[\mathbb{P}_{k}\right]=\left[X \oplus X \underset{\tau_{X}}{\stackrel{\mathrm{id}}{\longrightarrow}} X \oplus X\right]-\left[\mathbb{Q}_{k}\right] \in K_{0}(\Omega \mathcal{N}),
$$

where $X$ denotes the kernel of the first top differential of $\mathbb{P}_{k}$.

By the definition of the binary acyclic complex $\mathbb{P}_{k}$, we may choose

$$
X:=J_{k+1} \oplus \bigoplus_{n=1}^{k}\left(J_{n} \oplus K_{n}\right) .
$$


As in the proof of Proposition 3.6, since $J_{n}$ and $K_{n}$ represent the same class in $K_{0}$ for all $n$, we have by Lemma 2.11

$$
\begin{gathered}
{\left[\left(J_{n} \oplus K_{n}\right) \oplus\left(J_{n} \oplus K_{n}\right) \underset{\tau_{J_{n} \oplus K_{n}}}{\stackrel{\mathrm{id}}{\longrightarrow}}\left(J_{n} \oplus K_{n}\right) \oplus\left(J_{n} \oplus K_{n}\right)\right]} \\
=2 \cdot\left[J_{n} \oplus J_{n} \underset{\tau_{J_{n}}}{\stackrel{\mathrm{id}}{\longrightarrow}} J_{n} \oplus J_{n}\right]=0 .
\end{gathered}
$$

Therefore,

$$
\left[X \oplus X \underset{\tau_{X}}{\stackrel{\mathrm{id}}{\longrightarrow}} X \oplus X\right]=\left[J_{k+1} \oplus J_{k+1} \underset{\tau_{J_{k+1}}}{\stackrel{\mathrm{id}}{\longrightarrow}} J_{k+1} \oplus J_{k+1}\right] .
$$

Similarly,

$$
Y:=K_{k+1} \oplus \bigoplus_{n=1}^{k}\left(J_{n} \oplus K_{n}\right)
$$

is the kernel of the first bottom differential of $\mathbb{P}_{k}$. Note that the complement of $J_{k+1}$ in $X$ and the complement of $K_{k+1}$ in $Y$ are the same; let $Z$ denote that complement.

Unwinding the definition of $\mathbb{Q}_{k}$, we see that, up to automorphisms flipping the two copies of $Z$ in the three lowest degrees of $\mathbb{Q}_{k}, \mathbb{Q}_{k}$ coincides with the sum of $\mathbb{P}_{k+1}$ with some complexes in the image of the diagonal functor $\Delta$. Since, by (5.7), $\left[Z \oplus Z \underset{\tau_{Z}}{\stackrel{\text { id }}{\rightrightarrows}} Z \oplus Z\right]=0$, we see that $\left[\mathbb{Q}_{k}\right]=\left[\mathbb{P}_{k+1}\right]$. Hence,

$$
\left[\mathbb{P}_{k}\right]=\left[J_{k+1} \oplus J_{k+1} \underset{\tau_{J_{k+1}}}{\stackrel{\mathrm{id}}{\longrightarrow}} J_{k+1} \oplus J_{k+1}\right]-\left[\mathbb{P}_{k+1}\right] .
$$

The argument for $k$ even is completely analogous. Therefore, we have for every $k \geq 0$ the equation

$$
[\mathbb{P}]=x(\mathbb{P}, k):=(-1)^{k}\left[\mathbb{P}_{k}\right]+\sum_{n=1}^{k}\left[J_{n} \oplus J_{n} \underset{\tau_{J_{n}}}{\stackrel{\mathrm{id}}{\longrightarrow}} J_{n} \oplus J_{n}\right] .
$$

Proof of Theorem 1.1. Define a map $\Psi: K_{0}(\Omega \mathcal{N}) \rightarrow K_{1}^{N}(\mathcal{N})$ by the rule

$$
[\mathbb{P}] \mapsto x(\mathbb{P}, k(\mathbb{P})),
$$

where $k(\mathbb{P})$ is defined to be

$$
k(\mathbb{P}):=\min \left\{n \in \mathbb{N} \mid P_{n^{\prime}} \cong 0 \text { for all } n^{\prime}>n\right\} .
$$

We have to show that this is a well-defined homomorphism. By our definition of $k(\mathbb{P})$, the complex $\mathbb{P}_{k(\mathbb{P})}$ has length two.

Let $\mathbb{P}^{\prime} \longmapsto \mathbb{P} \rightarrow \mathbb{P}^{\prime \prime}$ be an exact sequence of binary acyclic complexes. Evidently, $x\left(\mathbb{P}^{\prime}, k(\mathbb{P})\right)+x\left(\mathbb{P}^{\prime \prime}, k(\mathbb{P})\right)=x(\mathbb{P}, k(\mathbb{P}))$. Note that $k\left(\mathbb{P}^{\prime}\right), k\left(\mathbb{P}^{\prime \prime}\right) \leq k(\mathbb{P})$ and at least one of $k\left(\mathbb{P}^{\prime}\right)$ and $k\left(\mathbb{P}^{\prime \prime}\right)$ equals $k(\mathbb{P})$. If $k\left(\mathbb{P}^{\prime}\right)=k(\mathbb{P})=k\left(\mathbb{P}^{\prime \prime}\right)$, we already have $\Psi\left(\left[\mathbb{P}^{\prime}\right]\right)+\Psi\left(\left[\mathbb{P}^{\prime \prime}\right]\right)=\Psi([\mathbb{P}])$. Suppose $k\left(\mathbb{P}^{\prime}\right)<k(\mathbb{P})$. Then $\mathbb{P}_{k(\mathbb{P})}$ arises from $\mathbb{P}_{k\left(\mathbb{P}^{\prime}\right)}$ by interchanging the role of top and bottom differential $k(\mathbb{P})-k\left(\mathbb{P}^{\prime}\right)$ times. Since interchanging the top and bottom differential results only in a change of sign $($ Lemma 5.3 $)$ and $J_{n}^{\prime} \cong 0$ for $n>k\left(\mathbb{P}^{\prime}\right)$, we have $x\left(\mathbb{P}^{\prime}, k\left(\mathbb{P}^{\prime}\right)\right)=x\left(\mathbb{P}^{\prime}, k(\mathbb{P})\right)$. The case $k\left(\mathbb{P}^{\prime \prime}\right)<k(\mathbb{P})$ is analogous.

Suppose now that $\mathbb{P}$ lies in the image of the diagonal functor $\Delta: C^{q} \mathcal{N} \rightarrow B^{q} \mathcal{N}$. Then we may choose $J_{n}=K_{n}$ for all $n$. In this case, the top and bottom differential 
of $\mathbb{P}_{k(\mathbb{P})}$ are isomorphic. However, the two differentials do not agree on the nose but only after flipping all appearing $K_{n}=J_{n}$. Since each one of these appears three times in $\mathbb{P}_{k(\mathbb{P})}$, applying the Nenashev relation we see that

$$
\left[\mathbb{P}_{k(\mathbb{P})}\right]=\sum_{n=1}^{k(\mathbb{P})}\left[J_{n} \oplus J_{n} \underset{\tau_{J_{n}}}{\stackrel{\mathrm{id}}{\longrightarrow}} J_{n} \oplus J_{n}\right] .
$$

Consequently, $\Psi([\mathbb{P}])=0$ by Lemma 2.11 This shows that $\Psi$ is a well-defined homomorphism $K_{0}(\Omega \mathcal{N}) \rightarrow K_{1}^{N}(\mathcal{N})$.

Our previous discussion implies that $\Phi \circ \Psi=\operatorname{id}_{K_{0}(\Omega \mathcal{N})}$. What is left to do is to show that $\Psi \circ \Phi=\operatorname{id}_{K_{1}^{N}(\mathcal{N})}$. To do so, it suffices to establish equation [5.6 in $K_{1}^{N}(\mathcal{N})$ for all binary acyclic complexes of length two. Let

$$
\mathbb{P}=P_{2} \underset{d_{2}^{\prime}}{\stackrel{d_{2}}{\rightrightarrows}} P_{1} \underset{d_{1}^{\prime}}{\stackrel{d_{1}}{\rightrightarrows}} P_{0}
$$

be a binary acyclic complex of length two. Then $\mathbb{P}_{1}$ is the binary acyclic complex

$$
P_{2} \oplus P_{2} \longleftrightarrow P_{2} \oplus P_{2} \oplus P_{2} \oplus P_{1} \longrightarrow P_{2} \oplus P_{2} \oplus P_{0}
$$

with the following top and bottom differentials:

$$
\begin{aligned}
& P_{2} \longrightarrow P_{2} \\
& P_{2} \longrightarrow P_{2} \\
& P_{2} \longrightarrow P_{2} \\
& P_{2} \stackrel{d_{2}^{\prime}}{\longrightarrow} P_{1} \stackrel{d_{1}^{\prime}}{\longrightarrow} P_{0}
\end{aligned}
$$

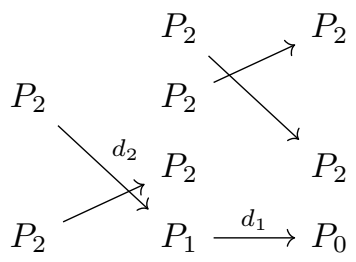

Consider the following binary acyclic double complex where the upper row is $\mathbb{P}_{1}$, the lower row is $\mathbb{P}$ with switched differentials plus $\Delta_{P_{2}} \oplus \Delta_{P_{2}} \oplus \Delta_{P_{2}}[1]$, and the middle vertical map $\tau_{P_{2}}$ denotes the flip of the second and third summand:

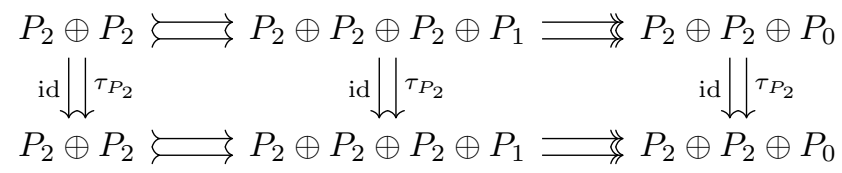

Therefore, we obtain using Lemmas 2.11 and 5.3

$$
[\mathbb{P}]=\left[P_{2} \oplus P_{2} \underset{\tau_{P_{2}}}{\stackrel{\mathrm{id}}{\longrightarrow}} P_{2} \oplus P_{2}\right]-\left[\mathbb{P}_{1}\right] .
$$

This finishes the proof.

Proof of Theorem 1.4. The statement is obtained by considering the first part of the proof of Theorem 1.1 and observing that Lemmas 5.3 and 2.11 and the Nenashev relation used there only require total complexes of length at most three.

\section{ACKNOWLEDGMENTS}

The authors are indebted to Daniel Grayson for sharing with us his proof of surjectivity of $\Phi$. We thank Robin Loose for helpful discussions and Bernhard Köck for useful comments on a previous version. 


\section{REFERENCES}

[Bar16] Clark Barwick, On the algebraic K-theory of higher categories, J. Topol. 9 (2016), no. 1, 245-347, DOI 10.1112/jtopol/jtv042. MR3465850

[BGT13] Andrew J. Blumberg, David Gepner, and Gonçalo Tabuada, A universal characterization of higher algebraic K-theory, Geom. Topol. 17 (2013), no. 2, 733-838, DOI 10.2140/gt.2013.17.733. MR3070515

[BR07] Arthur Bartels and David Rosenthal, On the K-theory of groups with finite asymptotic dimension, J. Reine Angew. Math. 612 (2007), 35-57, DOI 10.1515/CRELLE.2007.083. MR2364073

[Car95] Gunnar Carlsson, On the algebraic K-theory of infinite product categories, K-Theory 9 (1995), no. 4, 305-322, DOI 10.1007/BF00961467. MR1351941

[CP95] Gunnar Carlsson and Erik Kjær Pedersen, Controlled algebra and the Novikov conjectures for $K$ - and L-theory, Topology 34 (1995), no. 3, 731-758, DOI 10.1016/00409383(94)00033-H. MR 1341817

[Gra] Daniel R. Grayson, Relative algebraic K-theory by elementary means, arXiv:1310.8644.

[Gra12] Daniel R. Grayson, Algebraic K-theory via binary complexes, J. Amer. Math. Soc. 25 (2012), no. 4, 1149-1167, DOI 10.1090/S0894-0347-2012-00743-7. MR2947948

[GZ67] P. Gabriel and M. Zisman, Calculus of fractions and homotopy theory, Ergebnisse der Mathematik und ihrer Grenzgebiete, Band 35, Springer-Verlag New York, Inc., New York, 1967. MR0210125

[Har15] Thomas K. Harris, Binary complexes and algebraic K-theory, Ph.D. thesis, Southampton, 2015.

[Hel65] Alex Heller, Some exact sequences in algebraic K-theory, Topology 4 (1965), 389-408, DOI 10.1016/0040-9383(65)90004-2. MR179229

[Kas15] Daniel Kasprowski, On the K-theory of groups with finite decomposition complexity, Proc. Lond. Math. Soc. (3) 110 (2015), no. 3, 565-592, DOI 10.1112/plms/pdu062. MR3342098

[KW19] Daniel Kasprowski and Christoph Winges, Algebraic $k$-theory of stable $\infty$-categories via binary complexes, Journal of Topology 12 (2019), no. 2, 442-462.

[Nen98] A. Nenashev, $K_{1}$ by generators and relations, J. Pure Appl. Algebra 131 (1998), no. 2, 195-212, DOI 10.1016/S0022-4049(97)00056-X. MR.1637539

[Qui73] Daniel Quillen, Higher algebraic K-theory. I, Algebraic K-theory, I: Higher K-theories (Proc. Conf., Battelle Memorial Inst., Seattle, Wash., 1972), Springer, Berlin, 1973, pp. 85-147. Lecture Notes in Math., Vol. 341. MR0338129

[RTY14] Daniel A. Ramras, Romain Tessera, and Guoliang Yu, Finite decomposition complexity and the integral Novikov conjecture for higher algebraic K-theory, J. Reine Angew. Math. 694 (2014), 129-178, DOI 10.1515/crelle-2012-0112. MR3259041

[Sch04] Marco Schlichting, Delooping the K-theory of exact categories, Topology 43 (2004), no. 5, 1089-1103, DOI 10.1016/j.top.2004.01.005. MR.2079996

[Sch06] Marco Schlichting, Negative K-theory of derived categories, Math. Z. 253 (2006), no. 1, 97-134, DOI 10.1007/s00209-005-0889-3. MR2206639

[Tho97] R. W. Thomason, The classification of triangulated subcategories, Compositio Math. 105 (1997), no. 1, 1-27, DOI 10.1023/A:1017932514274. MR.1436741

Mathematisches Institut, Rheinische Friedrich-Wilhelms-Universität Bonn, EndeNiCher Allee 60, 53115 Bonn, Germany

Email address: kasprowski@uni-bonn.de

$U R L:$ http://www . math.uni-bonn.de/people/daniel/

Max-Planck-Institut für Mathematik, Vivatsgasse 7, 53111 Bonn, Germany

Email address: winges@mpim-bonn.mpg.de

URL: http://www.math.uni-bonn.de/people/winges/ 